\title{
EL TABÚ SEXUAL EN EL ESPAÑOL DE COSTA RICA: ANÁLISIS SOCIOLINGÜÍSTICO
}

\author{
Annette Calvo Shadid*
}

\begin{abstract}
RESUMEN
Este artículo presenta los resultados de un estudio sociolingüístico acerca del uso del léxico del tabú sexual en su variación diafásica en cuatro cantones de la provincia de San José, Costa Rica. Se trabaja con cinco variables sociolingüísticas: sexo, edad, nivel de instrucción, nivel socioeconómico y lugar de residencia. Sus resultados presentan un alto grado nivel de confianza, por haber sido sometidos a pruebas de significancia estadística.

Palabras clave: léxico, tabú sexual, sociolingüística, lingüística cuantitativa, español de Costa Rica.
\end{abstract}

\begin{abstract}
This article presents the results of a sociolinguistics study on the use of lexicon on sexual taboo in its diaphasic variability of Costa Rican Spanish, as perceived in four areas of San José, Costa Rica. It works with five sociolinguistics variables: sex, age, educational level, socioeconomic level, and place of residence. Results are highly reliable as they are supported by statistically significant proofs.

Key Words: lexicon, sexual taboo, sociolinguistics, quantitative linguistics, Costa Rican Spanish.
\end{abstract}

\section{Introducción}

Esta investigación constituye un estudio sociolingüístico de tipo cuantitativo sobre el supuesto uso del léxico del tabú sexual en cuatro cantones de la provincia de San José, Costa Rica. En el estudio se incluyen cinco variables sociolingüísticas: el sexo, la edad, el nivel de instrucción, el nivel socioeconómico y el lugar de residencia de los hablantes, además de que se toma en cuenta la variación diafásica o el estilo en que este tipo de léxico se manifiesta.

Para esto, se utilizan ocho variables situacionales: cuatro formales (Usted hablando en una entrevista de trabajo, Usted en una declaración frente a un tribunal de justicia; Usted hablando en una entrevista por televisión; Usted hablando en un congreso profesional o en una asamblea o reunión de trabajo) y cuatro informales (Usted hablando en broma con un amigo/a íntimo a solas; Usted hablando con su 
cónyuge o pareja asolas; Usted hablando conun grupo de amigos en su propia casa; Usted hablando con personas de confianza durante un juego de mesa). Estas ocho variables fueron probadas en un estudio piloto preliminar' ${ }^{1}$. Además de las situaciones, se toman en cuenta diez lexías tabuizadas referentes a los siguientes aspectos del ámbito de lo sexual:

a) Partes del cuerpo: picha, huevos, mico, tetas, culo

b) Actividad sexual: culear, regarse

c) Comportamiento sexual: puta, playo, tortillera

Por sus características, esta investigación constituye la descripción de un microestudio ${ }^{2}$, sobre la base del análisis cuantitativo de un léxico tabuizado de connotación sexual. Es de alcances sincrónico, sintópico, y exploratorio general de réplica ${ }^{3}$. Se realiza en un ambiente relativamente natural, por medio de entrevistas.

En el estudio se trabaja por comparación porcentual y con técnicas estadísticas para el análisis de la información tales como las distribuciones de frecuencia, cruce de variables y comparación de medias con base en el análisis de varianza.

\subsection{Objetivos}

\subsubsection{Objetivo general}

Realizar un análisis sociolingüístico cuantitativo del léxico sexual tabuizado en el español de Costa Rica.

\subsubsection{Objetivos específicos}

1. Tomar una muestra estratificada de léxico sexual tabuizado en cuatro cantones de la provincia de San José.

2. Realizar un análisis cuantitativo del léxico sexual tabuizado y las situaciones comunicativas planteadas, de acuerdo con el sexo, la edad, el nivel de instrucción, la situación socioeconómica y el lugar de residencia de los hablantes de cuatro cantones seleccionados de la provincia de San José.

\section{Diseño metodológico}

\subsection{Selección y características de la muestra}

La muestra para esta investigación se seleccionó tomando en consideración cuatro cantones de la Gran Área Metropolitana de la provincia de San José, uno al norte (Tibás), uno al sur (Alajuelita), uno al este (Montes de Oca), y uno al oeste (Santa Ana). Se eligieron las cabeceras de cantón, a saber, San Juan (Tibás), Distrito Central (Alajuelita), Sabanilla (Montes de Oca) y Distrito Central (Santa Ana). El tamaño de la muestra se calculó sobre la base del $0.05 \%$ de la población mayor de 25 años.

Las técnicas estadísticas utilizadas para el análisis de la información fueron las distribuciones de frecuencia, el cruce de variables y la comparación de medias con base en el análisis de varianza.

Para el procesamiento de los datos se diseñó una base de datos creada en Excel y el análisis estadístico de los datos se realizó en SPSS, versión 8.0, y en Excel.

El procedimiento de evaluación consistió en construir un índice de supuesto uso de las palabras tabú, con el fin de compararlas entre las diferentes situaciones (formales e informales), y por las características sociodemográficas (sexo, lugar de procedencia, edad, nivel educativo y nivel socioeconómico). Este indicador varía entre 0 y 100, donde 0 implica nada de uso y 100 uso máximo de las lexías en cuestión.

Con el objeto de clasificar a las personas según su nivel socioeconómico, se construyó un índice ponderado con respecto a la tenencia de una serie de artículos utilizados en la Encuesta de Hogares de Propósitos Múltiples del Instituto Nacional de Estadística y Censos. Posteriormente, se dividió el puntaje en quintiles, con el fin de ubicar a las personas según su nivel de ingresos. Se probó que el índice resultó apropiado para 
estimar la concentración de la población según los quintiles de ingreso observados en el Censo de Población del año 2000.

La muestra seleccionada permite estimaciones con un nivel de confianza del $95 \%$, un error máximo permisible del $6 \%$, y una variabilidad del $16 \%$, considerando el supuesto de homogeneidad de uso del lenguaje cercano al $80 \%$. Se ajustó el tamaño de la muestra a un $20 \%$ por no respuesta.

La fórmula para determinar el tamaño de la muestra para el estudio es la siguiente,

$$
n=\frac{\left(z_{(1-\alpha / 2)}\right) \sigma^{2}}{(d)^{2}}
$$

en la cual,

$n$ es el tamaño de muestra

$z_{(1-\alpha / 2)}$ es el valor asociado al nivel de confianza en la distribución normal estándar.

$d$ es el error máximo permisible .

$\sigma^{2}$ es la variabilidad estimada suponiendo que la población muestra una homogeneidad de uso del lenguaje cercano al $80 \%$. siguiente:

Sustituyendo en la fórmula resulta lo

$$
n=\frac{(1.96)^{2}}{(0.06)^{2}} 0.16=171
$$

Posteriormente, se hizo la corrección por finitud de la siguiente forma:

$$
\mathrm{n} f=\frac{\mathrm{n}}{1+\frac{\mathrm{n}}{\mathrm{N}}}=\frac{171}{1+\frac{171}{27401}} \approx 171
$$

$\mathrm{n}_{f}$ muestra final para cada grupo

$N$ Población total

Por lo tanto, la muestra seleccionada finalmente resultó, ajustando el tamaño final por un $20 \%$ de no respuesta, en un total propuesto de 205 personas; sin embargo, al final se entrevistaron 245 personas, lo cual favoreció el nivel de estimación de los parámetros.

Los individuos se escogieron mediante un muestreo de segmentos censales de cada uno de los distritos seleccionados, de los cuales se completó una muestra por cuota. Se consideraron las proporciones por edad y sexo encontradas en el Censo de Población de Costa Rica del año 2000.

\subsection{El instrumento}

Este estudio trata de recoger, por medio de encuesta, una muestra de vocabulario del tabú sexual respecto de lo que la población opina que usa o no usa, y sus posibles sustitutos léxicos.

Se utilizó un cuestionario con ocho situaciones comunicativas, a saber, cuatro formales y cuatro informales, y diez lexías que debían pasar por todas las situaciones, y expresar si las usarían o no las usarían en su habla. Si la respuesta era no, debían anotar cuál palabra en sustitución de esta usarían en cada contexto.

El cuestionario utilizado sigue a López Morales (1990) y se adaptó a la realidad social y lingüística de los costarricenses. Primero se probaron doce situaciones, de las cuales ocho de ellas se ubicaron claramente como formales e informales, cuatro de estilo informal y cuatro de estilo formal.

Las situaciones que en López Morales (1990) y en Martínez Valdueza (1995) se recogieron como neutrales no se registraron como tales en la población costarricense, según la preencuesta realizada por la investigadora de este proyecto.

Una vez definidas las situaciones, se estudiaron las palabras que podían percibirse como tabú dentro de la población costarricense en el ámbito sexual, haciendo varias consultas y entrevistas con distintas personas.

Se seleccionaron diez lexías relacionados con partes del cuerpo (picha, huevos, mico, tetas, culo), con actividad sexual (culear, regarse) y con comportamiento sexual puta, playo y tortillera). Debajo de cada una de ellas, junto a cada una de las situaciones, los encuestados debían llenar 
la casilla respectiva de Sí o No, dependiendo de si usaría la palabra tabú en las diferentes situaciones. Si la respuesta era No, debía anotar debajo de la palabra Usaría, en la línea continua, cuál otra palabra de significado semejante usaría en la situación determinada.

Se consultaron, en una misma hoja diseñada para tales efectos, los datos personales que permitieran ubicar posteriormente la muestra, como el sexo, la edad, el lugar de residencia, el lugar de nacimiento, el nivel educativo, y la tenencia de artículos y propiedades, como ya se ha mencionado.

En la primera página, se realizó una descripción del cuestionario y se proporcionaron las instrucciones sobre cómo llenarlo. Se colocaron dos casillas de consentimiento informado, las cuales los encuestados debían llenar.

En total, el cuestionario sumó siete hojas: la primera con la descripción, el consentimiento y las instrucciones, y luego, las hojas de respuestas 1, 2, 3, 4 y 5, contenían las situaciones y las palabras tabuizadas para llenar; la hoja de respuestas 6 contenía la información personal que debían completar.

\subsection{Las hipótesis estadísticas de trabajo}

La técnica de análisis de datos corresponde al análisis de varianza, como se ha señalado, con el fin de probar las siguientes hipótesis:

Ho: El porcentaje promedio de uso del lenguaje tabú es igual por sexo.

$\mathrm{H}_{1}$ : Al menos uno de los promedios es diferente.

Ho: El porcentaje promedio de uso del lenguaje tabú es igual por grupo de edad.

$\mathrm{H}_{1}$ : Al menos uno de los promedios es diferente.

Ho: El porcentaje promedio de uso del lenguaje tabú es igual por nivel educativo.

$\mathrm{H}_{1}$ : Al menos uno de los promedios es diferente.

Ho: El porcentaje promedio de uso del lenguaje tabú es igual por nivel socioeconómico.
$\mathrm{H}_{1}$ : Al menos uno de los promedios es diferente.

Ho: El porcentaje promedio de uso del lenguaje tabú es igual por lugar de residencia

$\mathrm{H}_{1}$ : Al menos uno de los promedios es diferente.

Ho: El porcentaje promedio de uso del lenguaje tabú es igual por situación comunicativa

$\mathrm{H}_{1}$ : Al menos uno de los promedios es diferente.

\subsection{Proyecto de campo}

El proyecto se realizó a manera de encuesta en las cabeceras de cantón seleccionadas. Las encuestas se aplicaron con la colaboración de siete personas entrenadas previamente, en estudio piloto, para este propósito. Se trataba de estudiantes de las carreras de Filología Española o de Filología Clásica, en su mayoría, y aplicaron el instrumento en un lapso de cuatro sesiones de trabajo durante el mes de agosto del 2006. El trabajo de campo fue supervisado constantemente por la investigadora del proyecto, por una estadística y por una experta en el área de la metodología de la investigación.

\section{Análisis cuantitativo de los resultados}

El procedimiento de evaluación en este trabajo consistió en construir un índice de uso de las palabras relacionadas con el tabú sexual, con el fin de compararlas con las diferentes situaciones (formales e informales), y con las características sociodemográficas (sexo, edad, nivel educativo, nivel socioeconómico y lugar de residencia).

El indicador varía entre 0 y 100, en el que 0 implica ningún uso y 100 un máximo de uso de las lexías tabuizadas.

\subsection{Análisis de la muestra}

La muestra seleccionada permitió estimaciones con un nivel de confianza del $95 \%$, un error máximo permisible del $6 \%$, y una 
variabilidad del 16\%, considerando el supuesto de homogeneidad de uso del lenguaje cercano al $80 \%$. Se ajustó el tamaño de la muestra a un $20 \%$ por no respuesta.

El total de personas entrevistadas fue de 245, distribuidas proporcionalmente en los cuatro distritos de San José, lo cual implica que las estimaciones realizadas en el estudio se ajustaban al 5\% de error máximo permisible.

Un $52,7 \%$ del total de los entrevistados son mujeres, y el $47,3 \%$ son hombres, en proporciones muy similares en todas las cabeceras de cantón.

Así, las proporciones observadas por sexo y lugar de residencia de la muestra no presentan diferencia estadísticamente significativa $(\mathrm{p}=0,12)$ con respecto a las proporciones observadas en la muestra planeada. Por ejemplo, en Alajuelita la muestra resultó en un total de $6,5 \%$ de hombres y un $7,8 \%$ de mujeres; en Sabanilla fue un total de $12,7 \%$ de hombres y un $9 \%$ de mujeres; en Santa Ana la muestra fue de $7,3 \%$ de hombres y de $8,6 \%$ de mujeres; en San Juan de Tibás, fue de un $24,5 \%$ de hombres y de $21,2 \%$ de mujeres.

Las proporciones por edad y sexo en la muestra también se mantuvieron como se esperaba. La edad promedio de los entrevistados fue de 42,0 años (IC 95\%: 40,2 - 43,8). No se encontró diferencia estadísticamente significativa por sexo $(\mathrm{p}=0,480)$. En los hombres, la edad promedio fue de 42,6 años, y la de las mujeres, de 41,3 años.

Respecto del nivel educativo, el número promedio de años de estudio fue de 10. El primer grupo de entrevistados, con primaria incompleta, mostró un porcentaje similar por sexo: $10,3 \%$ de hombres y $9,4 \%$ de mujeres. El segundo grupo, primaria completa, también mostró porcentajes semejantes: $16,4 \%$ de hombres y $17,1 \%$ de mujeres. El tercer grupo, con secundaria incompleta, mostró un $28,4 \%$ de hombres y un $26,9 \%$ de mujeres. El cuarto grupo, con secundaria completa, muestra un $13,8 \%$ de hombres y un $17,6 \%$ de mujeres. El quinto grupo, con formación universitaria incompleta, presentó un $24,1 \%$ de hombres y un $21,2 \%$ de mujeres. El sexto y último grupo, con formación universitaria completa, también presentó semejanza por sexo: 6,9\% de hombres y $7,8 \%$ de mujeres. Se observa una ligera mayoría de mujeres, no significativa, en los rubros de primaria, secundaria y universitaria completas.

Con respecto al nivel socioeconómico de la población, se encontró que existe una mayor concentración de población en los quintiles más bajos en los cantones de Alajuelita y Santa Ana, mientras que en los cantones de Sabanilla y Tibás se concentró la población en los quintiles más altos de ingresos. En los dos primeros quintiles de ingreso, los cuales registran un nivel socioeconómico bajo, se concentró más del $50 \%$ de la muestra entrevistada en Alajuelita y en Santa Ana. Por el contrario, en Sabanilla y en Tibás, se fue alcanzando el 50\% de la muestra entrevistada entre los quintiles III y IV; es decir, entre los niveles medio y medio alto. Lo anterior muestra que la variable lugar de residencia podría incidir en los resultados de la muestra recogida.

\subsection{Análisis de uso de las lexías}

El uso de las lexías varía según se trate del tipo de categoría a que se refiera: el léxico tabuizado que se relaciona con las partes del cuerpo (picha, huevos y mico) y con las acciones de tipo sexual (culear, regarse) se usa menos, mientras el relacionado con el comportamiento sexual se utiliza con mayor frecuencia (playo, tortillera y puta). Esto podría indicar que para las personas es más tabú hablar de lo que ellas mismas tienen o hacen, que juzgar el comportamiento social de los otros, sobre todo si esta está estigmatizado en alguna medida.

En términos generales, el promedio de uso del tabú en esta investigación es del 51,4\% (IC95\%: 46,6 - 56,1).

Los porcentajes de uso se pueden apreciar en el siguiente gráfico. En él, las lexías se han organizado de menor a mayor uso, empezando por las partes del cuerpo picha y mico, que precisamente corresponden a los genitales masculino y femenino; luego las actividades sexuales culear y regarse; luego otras partes del cuerpo menos tabuizadas, huevos, culo y tetas y, por último, las menos tabuizadas, 
relacionadas con el comportamiento sexual puta, tortillera y playo.

Se puede observar que la lexía menos usada en términos generales, es supuestamente picha (46\%), seguida de cerca por mico $(47,1 \%)$ y de culear $(47,8 \%)$. Las lexías regarse $(49 \%)$ y huevos $(49,1 \%)$ presentan un uso un tanto mayor, y llegan casi al $50 \%$ de uso. Las que sobrepasan un uso del $50 \%$ son culo $(52,3 \%)$, tetas $(53,3 \%)$, y las tres relacionadas con el comportamiento sexual, a saber, puta $(53,8 \%)$, tortillera $(56,4 \%)$ y playo (59\%); esta última es la más usada.

Las lexías más usadas aparentemente para las partes del cuerpo son tetas $(53,3 \%)$ y culo $(52,3$. Asimismo, entre las que corresponden al comportamiento sexual, la lexía puta es la usada en menor porcentaje $(53,8 \%)$.

Respecto de la lexía menos usada de todas, supuestamente, picha, se registra un no uso de ella en un $56 \%$ entre las mujeres y un 38\% en los hombres.

GRÁFICO 1

Porcentaje de uso de lexías. San José: 2006.

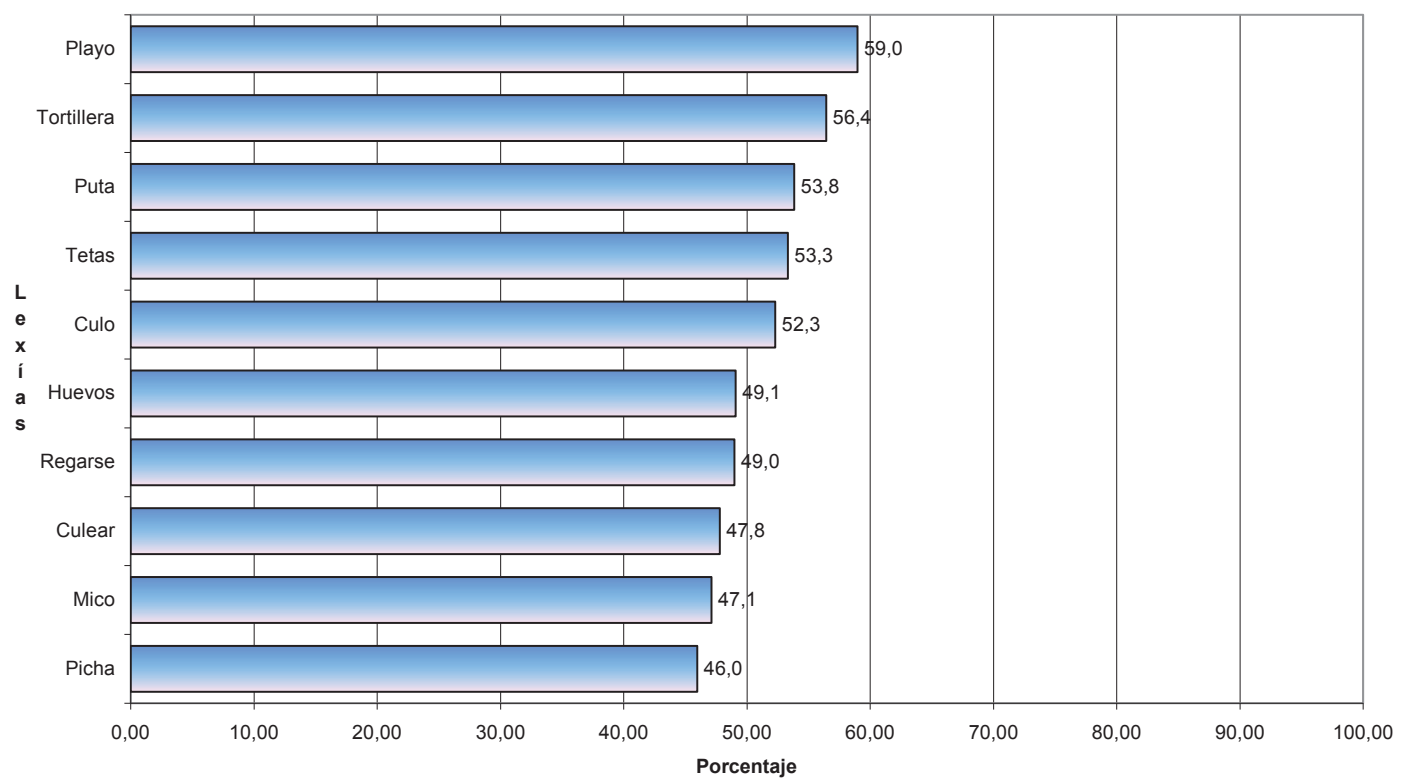

\subsection{Análisis de lexías por estilo (situaciones formales e informales)}

Como se ha señalado en el apartado anterior, el promedio de uso del tabú en esta investigación es del 51,4\% (IC 95\%: 46,6 - 56,1). En la totalidad de la muestra, se encontró diferencia estadísticamente significativa en el uso del tabú por grupos de edad $(p=0,044)$, lugar de residencia $(p=0,000)$ y nivel educativo $(p=0,009)$. No se proporcionó diferencia significativa en el uso ni por sexo ni por quintil de ingreso.
Se encontró diferencia estadísticamente significativa en el uso del tabú, además, si se trataba del estilo, es decir, entre situaciones formales e informales, ya que la diferencia de uso fue cerca del $22 \%$. En las situaciones formales su uso fue del 40,02\% (IC95\%: 34.74 - 45.30), mientras que en las situaciones informales el porcentaje de uso fue del 62,75\% (IC95\%: 57,84 $-67,65)$.

Respecto del estilo formal, se encontró diferencia estadísticamente significativa en el uso del tabú según el lugar de residencia $(\mathrm{p}=0,041)$, mientras que en el estilo informal se encontró diferencia estadísticamente significativa por 
lugar de residencia $(\mathrm{p}=0,000)$, grupos de edad $(p=0,001)$ y nivel educativo $(p=0,007)$; por sexo $\mathrm{y}$ por quintil de ingreso no se dio diferencia significativa en ninguno de los estilos. El siguiente cuadro muestra la significancia total de uso de las lexías tabuizadas según variable sociodemográfica por estilo.

El cuadro muestra que existe diferencia estadísticamente significativa en los dos estilos por lugar de residencia (formal $(p=0,041)$ e informal $(p=0,000)$.

Los porcentajes de uso del tabú por lugar de residencia según estilo, permiten observar que la diferencia significativa se produce en Alajuelita respecto de los otros cantones. En Alajuelita se presenta un uso general del tabú del $25,11 \%$, mientras que en Sabanilla este asciende significativamente a $52,81 \%$, en Santa Ana al 53,08\% y el mayor uso se da en Tibás, con $60,19 \%$.

En Alajuelita no se muestra una diferencia importante por el estilo: solamente hay un $4,21 \%$ de diferencia entre las situaciones formales $(23,00 \%)$ y las informales $(27,21 \%)$, mientras que en los otros cantones, por ejemplo en Sabanilla, existe una diferencia porcentual de

\begin{tabular}{lccc}
\hline \multicolumn{5}{c}{$\begin{array}{c}\text { CUADRO 1 } \\
\text { Significancia estadística de uso total de lexía según } \\
\text { variable sociodemográfica por estilo }\end{array}$} \\
\hline \multicolumn{2}{c}{ Total } & Formal & Informal \\
\hline Sexo & 0,184 & 0,421 & 0,089 \\
\hline Lugar de residencia & 0,000 & 0,041 & 0,000 \\
\hline Grupos de edad & 0,044 & 0,412 & 0,001 \\
\hline Nivel educativo & 0,009 & 0,045 & 0,007 \\
\hline Quintil & 0,816 & 0,872 & 0,800 \\
\hline
\end{tabular}

CUADRO 2

Porcentaje de uso de lexías según lugar de residencia por estilo

\begin{tabular}{|c|c|c|c|}
\hline \multirow[b]{2}{*}{ Lugar de residencia } & \multirow[b]{2}{*}{ Total } & \multicolumn{2}{|c|}{ Estilo } \\
\hline & & Formal & Informal \\
\hline Alajuelita & 25,11 & 23,00 & 27,21 \\
\hline Sabanilla & 52,81 & 39,72 & 65,90 \\
\hline Santa Ana & 53,08 & 41,67 & 64,49 \\
\hline Tibás & 60,19 & 46,16 & 74,22 \\
\hline Total & 51,38 & 40,02 & 62,74 \\
\hline
\end{tabular}

uso por estilo del $26,18 \%$ (39,72\% en situaciones formales y $65,90 \%$ en informales); en Santa Ana existe una diferencia porcentual de uso por estilo de 22,82\% (41,67\% en situaciones formales y $64,49 \%$ en informales); en Tibás existe una diferencia porcentual de uso por estilo del $28,06 \%$ (46,16\% en situaciones formales y $74,22 \%$ en informales). Se observa, de este 
modo, una diferencia general del 22,72\% por estilo según lugar de residencia.

La diferencia en el uso del tabú encontrada por grupos de edad es significativa estadísticamente solo en el estilo informal $(p=0,001)$. Esta diferencia se explica por la diferencia encontrada en el promedio del menor uso del tabú en el grupo de 55 años y más con respecto a los otros grupos de edad.

Mientras el promedio general de uso del tabú en la generación de 45-54 años es de un $57,91 \%$, el del grupo de 55 años y más es de $37,77 \%$. Los otros dos grupos presentan también un porcentaje bastante alto de uso de las lexías tabuizadas: en segundo lugar, el grupo de menos de 35 años presentó un uso del 53,99\%, y luego, el de $35-44$, un porcentaje de 53,33\%.

Mientras en el estilo formal el grupo mayor de edad presenta un uso del 32,66\%, el de 45-54 años lo presenta en un 47,62\%. También se mantiene el grupo de menos de 35 con el segundo lugar de uso, con un $40,66 \%$, y luego el segundo grupo, de 35 a 44 años, con un $39,49 \%$ de uso del tabú.

En el estilo informal, la diferencia significativa se da entre el grupo de edad de 55 años y más y el resto de los grupos: un $42,87 \%$ de uso, mientras el grupo de 45-54 muestra un uso del 68,20\%. Del mismo modo, el primer grupo de menos de 35 años con presenta un uso del $67,33 \%$, y el de $35-44$ presenta un porcentaje muy similar, $67,17 \%$.

Si se observa la diferencia porcentual por grupos de edad entre los dos estilos, también se observa un porcentaje alto de diferencia de uso del tabú, excepto en el grupo de 55 años y más, en el que la diferencia es solamente de un $10,21 \%$; en el de 45-55, la diferencia es del 20,58\%; en el de 35-44 años la diferencia es del 27,68\% y, en el de menos de 35 años, la diferencia es de $26,67 \%$.

El cuadro 3 muestra los porcentajes utilizados según grupos de edad por estilo.

Respecto del estilo por nivel educativo, se muestra una tendencia a un mayor uso de lexías conforme aumenta el nivel educativo, tanto en situaciones formales como informales. Sin embargo, solamente en el estilo informal se observa diferencia significativa $(p=0,007)$. En general, se observa un uso menor del tabú en los entrevistados con primaria incompleta en ambos estilos (34,02\%), luego en los de primaria completa (40,92\% en ambos estilos). A partir de los entrevistados con estudios de secundaria, el uso del tabú aumenta considerablemente en ambos estilos: secundaria incompleta $(51,16 \%)$ y completa $(56,08 \%)$. En universitaria incompleta, se observa el mayor uso del tabú en ambos estilos $(64,45 \%)$, y luego disminuye un tanto el uso en universitaria completa, principalmente en el estilo formal $(40,02 \%)$, con un uso total del 49,93\%.

Respecto del estilo informal, en el que se encontró diferencia significativa, se observa un porcentaje de uso menor en los niveles educativos más bajos, primaria incompleta $(42,72 \%)$ y primaria completa $(51,37 \%)$. A partir de secundaria incompleta, de nuevo aumentan los porcentajes de uso, $63,98 \%$ y $67,44 \%$ para la

CUADRO 3

Porcentaje de uso de lexías según grupo de edad por estilo

\begin{tabular}{llll}
\hline \multicolumn{1}{c}{ Trupos de edad } & \multicolumn{2}{c}{ Formal } & Informal \\
\hline \multicolumn{1}{c}{ Total } & & \\
\hline Menos de 35 & 53,99 & 40,66 & 67,33 \\
\hline $35-44$ & 53,33 & 39,49 & 67,17 \\
\hline $45-54$ & 57,91 & 47,62 & 68,20 \\
\hline 55 y más & 37,77 & 32,66 & 42,87 \\
\hline Total & 51,38 & 40,02 & 62,75 \\
\hline
\end{tabular}


secundaria completa, y llegan a un máximo de uso del $75,24 \%$ en la universitaria incompleta, para descender luego un tanto, de nuevo, en la universitaria completa $(63,03 \%)$.

En el estilo formal, aunque no hubo diferencia estadísticamente significativa, también se puede notar un incremento del tabú conforme aumenta el nivel educativo. Los niveles de primaria, incompleta $(25,33 \%)$ y completa $(30,48 \%)$, contemplan un uso muy bajo del tabú. Luego continúa aumentando en la secundaria incompleta $(38,33 \%)$ y en la completa $(44,71 \%)$ para llegar al uso máximo del tabú en el estilo formal en la universitaria incompleta $(53,65 \%)$, y descender un tanto, nuevamente, en la universitaria completa $(36,84 \%)$.

Respecto de la diferencia entre ambos estilos por nivel educativo, esta se mantiene en los mismos términos: el que muestra una menor diferencia es el nivel de primaria: incompleta
$(17,39 \%)$, completa $(20,89 \%)$; continúa en aumento la diferencia en la secundaria incompleta $(25,65 \%)$. Posteriormente, empieza a disminuir un tanto la diferencia entre ambos estilos en la secundaria completa $(22,73 \%)$, y más aún en la universitaria incompleta (21,59\%). Luego, en la universitaria completa, la diferencia se acentúa hasta llegar a un máximo de 26,19\%.

Lo anterior indica que los niveles que presentan un mayor uso del tabú, como secundaria completa y universitaria incompleta, a su vez, presentan una diferencia menor de uso del tabú en ambos estilos.

El siguiente cuadro muestra los porcentajes utilizados según nivel educativo por estilo.

Respecto del estilo según sexo, aunque las mujeres presentan un menor porcentaje de uso del tabú, tanto a nivel formal como informal, estas no presentaron diferencias estadísticamente significativas.

\begin{tabular}{lccc}
\hline \multicolumn{5}{c}{ CUADRO 4 } \\
Porcentaje de uso de lexías según nivel educativo por estilo \\
\hline \multicolumn{1}{c}{ Total } & Formal & Estilo \\
\hline Nivel educativo & & & \\
\hline \multicolumn{1}{c}{ Informal } \\
\hline Primaria incompleta & 34,02 & 25,33 & 42,72 \\
\hline Primaria completa & 40,92 & 30,48 & 51,37 \\
\hline Secundaria incompleta & 51,16 & 38,33 & 63,98 \\
\hline Secundaria completa & 56,08 & 44,71 & 67,44 \\
\hline Universitaria incompleta & 64,45 & 53,65 & 75,24 \\
\hline Universitaria completa & 49,93 & 36,84 & 63,03 \\
\hline Total & 51,38 & 40,02 & 62,74 \\
\hline
\end{tabular}

CUADRO 5

Porcentaje de uso de lexías según sexo por estilo

\begin{tabular}{lccc}
\hline & & \multicolumn{2}{c}{ Estilo } \\
\hline Sexo & Total & Formal & Informal \\
\hline Masculino & 54,42 & 42,07 & 66,76 \\
\hline Femenino & 48,01 & 37,74 & 58,28 \\
\hline Total & 51,38 & 40,02 & 62,74 \\
\hline
\end{tabular}


Respecto del estilo según quintil de ingreso, se encontró un porcentaje de uso del tabú muy similar a lo largo de los quintiles, y se muestra siempre el mismo comportamiento tanto en situaciones informales como formales.
Aunque no hubo diferencia significativa, los mayores porcentajes de uso del tabú se dan en los quintiles de ingreso más altos, el IV $(55,64 \%)$ y el V $(54,40 \%)$.

\begin{tabular}{lcccc}
\hline \multicolumn{5}{c}{ CUADRO 6 } \\
\multicolumn{4}{c}{ Porcentaje de uso de lexías según quintil de ingreso por estilo } \\
\hline Quintil de ingreso & Total & \multicolumn{3}{c}{ Estilo } \\
\hline I & 49,34 & 39,36 & 59,31 \\
\hline II & 49,79 & 37,71 & 61,88 \\
\hline III & 47,91 & 36,17 & 59,64 \\
\hline IV & 55,64 & 44,26 & 67,02 \\
\hline V & 54,40 & 42,70 & 66,10 \\
\hline Total & 51,38 & 40,02 & 62,74 \\
\hline
\end{tabular}

Respecto de cada una de las situaciones comunicativas, el siguiente cuadro muestra la relación existente entre las situaciones comunicativas y las lexías usadas en la investigación. Así, los porcentajes son los siguientes: Como ya se ha señalado, se encontró diferencia estadísticamente significativa en el uso del tabú por el estilo entre las situaciones formales e informales.

La relación entre las dos variables lingüísticas, a saber, lexías por estilo, dio como resultado que el uso de las palabras tabuizadas es mayor en las situaciones informales (62,75\%): 1 . Usted hablando en broma con un amigo íntimo

CUADRO 7

Porcentaje de uso de lexía según variable sociodemográfica por situación

\begin{tabular}{lllllllll}
\hline \multicolumn{1}{c}{ Lexía } & \multicolumn{7}{c}{ Situación } \\
\hline & 1 & 2 & 4 & 5 & 6 & 7 & 8 & \\
\hline & & & & & & & \\
picha & 61,6 & 52,7 & 51,0 & 37,6 & 35,9 & 35,1 & 56,7 & 37,1 \\
\hline huevos & 64,1 & 57,6 & 55,9 & 37,1 & 39,6 & 37,1 & 61,6 & 39,6 \\
\hline mico & 60,8 & 54,3 & 54,7 & 38,8 & 39,2 & 36,3 & 56,7 & 36,3 \\
\hline tetas & 69,8 & 64,5 & 63,7 & 38,4 & 43,3 & 40,8 & 66,1 & 40,0 \\
\hline culo & 69,8 & 61,6 & 63,3 & 42,0 & 41,2 & 38,4 & 61,2 & 40,8 \\
\hline culear & 64,1 & 58,4 & 56,3 & 38,4 & 38,0 & 34,7 & 55,9 & 36,7 \\
\hline regarse & 61,6 & 62,4 & 55,9 & 38,8 & 38,8 & 38,0 & 58,0 & 38,4 \\
\hline puta & 71,4 & 63,3 & 62,9 & 42,0 & 42,9 & 40,4 & 65,3 & 42,4 \\
\hline playo & 78,0 & 66,9 & 72,2 & 46,5 & 47,3 & 45,7 & 71,0 & 44,1 \\
\hline tortillera & 74,7 & 67,3 & 69,8 & 45,7 & 43,7 & 42,0 & 66,5 & 41,6 \\
\hline
\end{tabular}


a solas, 2. Usted hablando con su cónyuge o pareja a solas, 3. Usted hablando con un grupo de amigos en su propia casa, 7. Usted hablando con personas de confianza durante un juego de mesa.

Asimismo, el uso de las palabras tabuizadas es menor en todas las situaciones consideradas formales $(40,02 \%)$ : 4 . usted en una entrevista de trabajo, 5. usted en una declaración frente a un tribunal de justicia, 6 . usted en una entrevista por televisión y 8 . usted en un congreso profesional o asamblea o reunión de trabajo.

Respecto de las situaciones informales, en la situación 1. Usted hablando en broma con un amigo íntimo a solas, la lexía más utilizada por situación es playo, (78\%). Además, entre todas las situaciones, en esta se presenta la lexía playo con la más alta frecuencia de uso (78\%); la segunda lexía más usada en esta situación es tortillera $(74,7 \%)$; la tercera lexía más usada es puta $(71,4 \%)$. En cuarto lugar, las lexías más usadas son tetas y culo ( $68,9 \%$ cada una). En quinto lugar se usan huevos y culear $(64,1 \%$ cada una); en sexto lugar, picha y regarse $(61,6 \%$ cada una) y, en último lugar, mico $(60,8 \%)$.

Los resultados son muy semejantes a los anteriores, relacionados con el uso de las lexías por porcentaje. Son idénticos en los cinco primeros lugares, a saber, playo, puta, tortillera, tetas, solo que esta vez la lexía culo se igualó en porcentaje al uso a tetas; huevos y culear se igualan en porcentajes por situación. Sin embargo, presentan un porcentaje de diferencia distinto en el uso de lexías por porcentaje, pues culear se usa menos. Luego, los lugares sexto y séptimo son un tanto distintos, pero picha y mico, son, igualmente, los menos usados tanto en los porcentajes generales de uso por lexía como por estos, de lexía por situación.

Respecto de la situación 2. Usted hablando con su cónyuge o pareja a solas, los resultados son semejantes, pero no tan cercanamente como los de la situación 1 y el uso general de lexías. Presenta algunas ligeras variaciones. En primero y segundo lugar están, tortillera (67,3\%); playo $(66,9 \%)$. De nuevo esas dos lexías están en los dos primeros lugares pero, en este caso, no en mismo orden de las anteriores; en tercer lugar, en este caso, se ubica tetas (64,5\%); en cuarto lugar, puta $(63,3 \%)$; en quinto lugar, regarse $(62,4 \%)$; en sexto lugar, culo $(61,6 \%)$; en séptimo lugar, culear $(58,4 \%)$; en octavo lugar, huevos $(57,6 \%)$; en noveno lugar, mico $(54,3 \%)$; en décimo lugar, picha $(52,7 \%)$. Como en el caso del uso general de lexías, mico y picha se encuentran en los últimos lugares de uso.

Respecto de la situación 3. Usted hablando con un grupo de amigos en su propia casa, los resultados también son semejantes, con algunas ligeras variaciones. En primero y segundo lugar están, como en los casos por uso de lexía y por situación 1, playo $(72,2 \%)$ y tortillera $(69,8 \%)$; en tercer lugar, como en la situación 2 , se ubica tetas $(63,7 \%)$; en cuarto lugar, culo $(63,3 \%)$; en quinto lugar, puta $(62,9 \%)$; en sexto lugar, culear (56,3\%); en séptimo lugar, regarse $(55,9 \%)$, y huevos $(55,9 \%)$, en octavo lugar, mico $(54,7 \%)$; en noveno lugar, picha $(51,0 \%)$. Como en el caso del uso general de lexías, mico y picha se encuentran en los últimos lugares de uso en esta situación.

Respecto de la situación 7. Usted hablando con personas de confianza durante un juego de mesa, última de las situaciones informales, los resultados también son semejantes, con algunas ligeras variaciones. En primero y segundo lugar están, como en los casos por uso de lexía y por situación 1 y 3 , playo $(71,0 \%)$ y tortillera $(66,58 \%)$; en tercer lugar, como en la situaciones 2 y 3 , se ubica tetas $(66,1 \%)$; en cuarto lugar, como en la situación 2 , puta $(65,3 \%)$; en quinto lugar, huevos $(61,6 \%) 6$; en sexto lugar, culo $(61,2 \%)$ culear $(56,3 \%)$; en séptimo lugar, como en el caso del uso general de lexía y por situación 3 , regarse $(58,0 \%)$; en octavo lugar, como en el caso del uso de lexía, culear $(55,9 \%)$; en noveno lugar, picha y mico $(56,7 \%)$, como en el caso del uso general de lexía y en situaciones 2 y 3 .

En este apartado se han presentado las lexías por situación, y se ha observado cuál ha sido la frecuencia de cada una de ellas en cada tipo de situación. Los resultados totales de la relación de estas dos variables se presentan en el 
siguiente cuadro, que muestra los porcentajes e intervalos de confianza de cada una de las lexías por cada una de las situaciones.

Como se puede observar en el cuadro, se muestra el intervalo de confianza de la muestra, que es del $95 \%$, y se proporciona el porcentaje de uso de las lexías por cada situación dentro de los límites inferior y superior del intervalo de confianza.

El mayor porcentaje de uso de las lexías se da en la situación 1. Usted hablando en broma con un amigo íntimo a solas, $(62,3 \%)$; en segundo lugar, en la situación 7. usted hablando con personas de confianza durante un juego de mesa, (61,9\%); en tercer lugar, en la situación 2. Usted hablando con su cónyuge o pareja a solas, $(60,9 \%)$; en cuarto lugar, en la situación 3. Usted hablando con un grupo de amigos en su propia casa, (60,6\%); en quinto lugar, en la situación 5. usted en una declaración frente a un tribunal de justicia, (41,0\%); en sexto lugar, en la situación 4. usted en una entrevista de trabajo, $(40,5 \%)$; en séptimo lugar, en la situación 8 . usted en un congreso profesional o asamblea o reunión de

CUADRO 8

Porcentaje e intervalo de confianza del uso de lexías según situación comunicativa

\begin{tabular}{llll}
\hline & $\begin{array}{c}\text { Porcentaje de } \\
\text { uso }\end{array}$ & \multicolumn{1}{l}{ Intervalo de confianza del 95\% } \\
\hline Situación & & Límite Inferior & Límite Superior \\
\hline 1. En broma con un amigo íntimo a solas & 62,3 & 62,6 & 72,6 \\
\hline 2. Con su cónyuge o pareja a solas & 60,9 & 55,7 & 66,1 \\
\hline 3. Con grupo de amigos en su propia casa & 60,6 & 55,5 & 65,7 \\
\hline 4. Entrevista de trabajo & 40,5 & 35,1 & 45,9 \\
\hline 5. En declaración frente a un tribunal de justicia & 41,0 & 35,6 & 46,3 \\
\hline 6. En entrevista en televisión & 38,9 & 33,4 & 44,3 \\
\hline 7. Con personas de confianza durante un juego de mesa & 61,9 & 56,7 & 67,1 \\
\hline 8. En congreso profesional o asamblea o reunión de trabajo & 39,7 & 34,3 & 45,2 \\
\hline
\end{tabular}

trabajo, (39,7\%) y, en octavo lugar, en la situación 6. usted en una entrevista por televisión (38,9\%).

En el cuadro 9 se pueden apreciar los porcentajes de las lexías tabuizadas por las situaciones en orden de mayor a menor uso.

Como se puede observar, la situación considerada menos formal es la $1(62,3 \%$ de uso), y la más formal, la 6 . (38,9\% de uso). Entre ellas, hay una diferencia porcentual de cerca del $23,4 \%$.

\subsection{Análisis de lexías por variables sociodemográficas}

La aplicación de la prueba de significancia estadística proporcionó los siguientes resultados:
El análisis del cuadro 10 indica que las frecuencias de uso de lexía por sexo no proporcionaron diferencia significativa en ninguna de las lexías.

El siguiente gráfico muestra, por medio de barras, las diferencias porcentuales por sexo.

En el gráfico se observa claramente la diferencia no significativa por sexo; en los hombres, sin embargo, se observa un mayor uso de las lexías que en las mujeres.

Por sexo, la lexía más usada por ambos sexos es playo $(59,0 \%)$, seguido por tortillera $(56,4 \%)$, puta $(53,8 \%)$, tetas $(53,3 \%)$, culo $(52,3 \%)$, huevos y regarse $(49,1 \%)$, culear $(47,8 \%)$, mico $(47,1 \%)$ y picha $(46,0 \%)$. Es decir, claramente se usan más las lexías referidas al comportamiento sexual, luego las de ciertas partes del cuerpo y 


\begin{tabular}{lc}
\hline \multicolumn{2}{c}{$\begin{array}{c}\text { CUADRO 9 } \\
\text { Ordenamiento de situaciones según porcentaje de uso de lexías }\end{array}$} \\
\hline \multicolumn{1}{c}{ Situación } & $\begin{array}{c}\text { Porcentaje } \\
\text { de uso }\end{array}$ \\
\hline 1. En broma con un amigo íntimo a solas & 62,3 \\
\hline 7. Con personas de confianza durante un juego de mesa & 61,9 \\
\hline 2. Con su cónyuge o pareja a solas & 60,9 \\
\hline 3. Con grupo de amigos en su propia casa & 60,6 \\
\hline 5. En declaración frente a un tribunal de justicia & 41,0 \\
\hline 4. Entrevista de trabajo & 40,5 \\
\hline 8. En congreso profesional o asamblea o reunión de trabajo & 39,7 \\
\hline 6. En entrevista en televisión & 38,9 \\
\hline
\end{tabular}

actividad sexual. Las dos lexías menos usadas son, precisamente, las de las partes del cuerpo más tabuizadas, mico y picha.

Respecto de la variable lugar de residencia, se ha encontrado diferencia significativa en todas las lexías: picha $(\mathrm{p}=0,001)$; huevos ( $\mathrm{p}$ $=0,000)$; mico $(\mathrm{p}=0,008)$; tetas $(\mathrm{p}=0,000)$; culo $(\mathrm{p}=0,000)$; culear $(\mathrm{p}=0,001)$; regarse $(\mathrm{p}$ $=0,000)$; puta $(\mathrm{p}=0,000)$; playo $(\mathrm{p}=0,000)$; tortillera $(\mathrm{p}=0,000)$.

Se observa que Alajuelita presenta un uso significativamente menor de lexías tabuizadas respecto de los otros cantones. Alajuelita es uno de los cantones más tradicionales de San José 4 . El centro del cantón está representado por un elevado número de familias arraigadas allí desde hace muchas décadas. Es probable que esta característica incidiera en un uso menor del tabú que los otros cantones analizados. Por otra parte, se encontró que existe una mayor concentración de población en los quintiles más bajos en los cantones de Alajuelita y Santa Ana, especialmente en Alajuelita. Podría ser que esta característica también incidiera en el menor uso de las lexías. A mayor concentración en los quintiles más bajos de población, menor es el uso del tabú.

El siguiente gráfico muestra las diferencias porcentuales por lugar de residencia.

En el gráfico se observa claramente la diferencia significativa por lugar de residencia, en el cual el cantón de Alajuelita exhibe un uso significativamente menor de todas las lexías en general. El cantón que exhibe una frecuencia mayor de uso del tabú es Tibás, con excepción de la lexía puta, que es más frecuente en Santa Ana. Luego, Santa Ana, donde son más frecuentes que en Sabanilla puta, playo y tortillera. Después le sigue muy de cerca Sabanilla, con cinco lexías más frecuentemente que en Santa Ana, picha, huevos, mico, tetas y culear. La lexía regarse, aparece prácticamente con el mismo porcentaje en Santa Ana (48,7\%) y en Sabanilla $(48,6 \%)$.

Así como Alajuelita es el cantón que representa la mayor concentración de población en los primeros quintiles de ingresos, y Tibás el que presenta la mayor concentración en los quintiles más altos, igualmente en ellos se da tanto el menor como el mayor uso de lexías tabuizadas.

La variable edad ha presentado diferencia significativa en las lexías huevos $(p=0,020)$, culear $(\mathrm{p}=0,016)$ y regarse $(\mathrm{p}=0,004)$.

Las lexías huevos, culear y regarse, presentan todas porcentajes de uso mucho más bajos en el grupo mayor de 55 años y más. Es decir, estas personas han evitado con mayor frecuencia el uso de las lexías relacionadas con actividad sexual y con una de las partes del cuerpo. En una de las encuestas, una mujer de este grupo de edad dijo desconocer del todo el significado de las lexías culear y regarse. Por este 


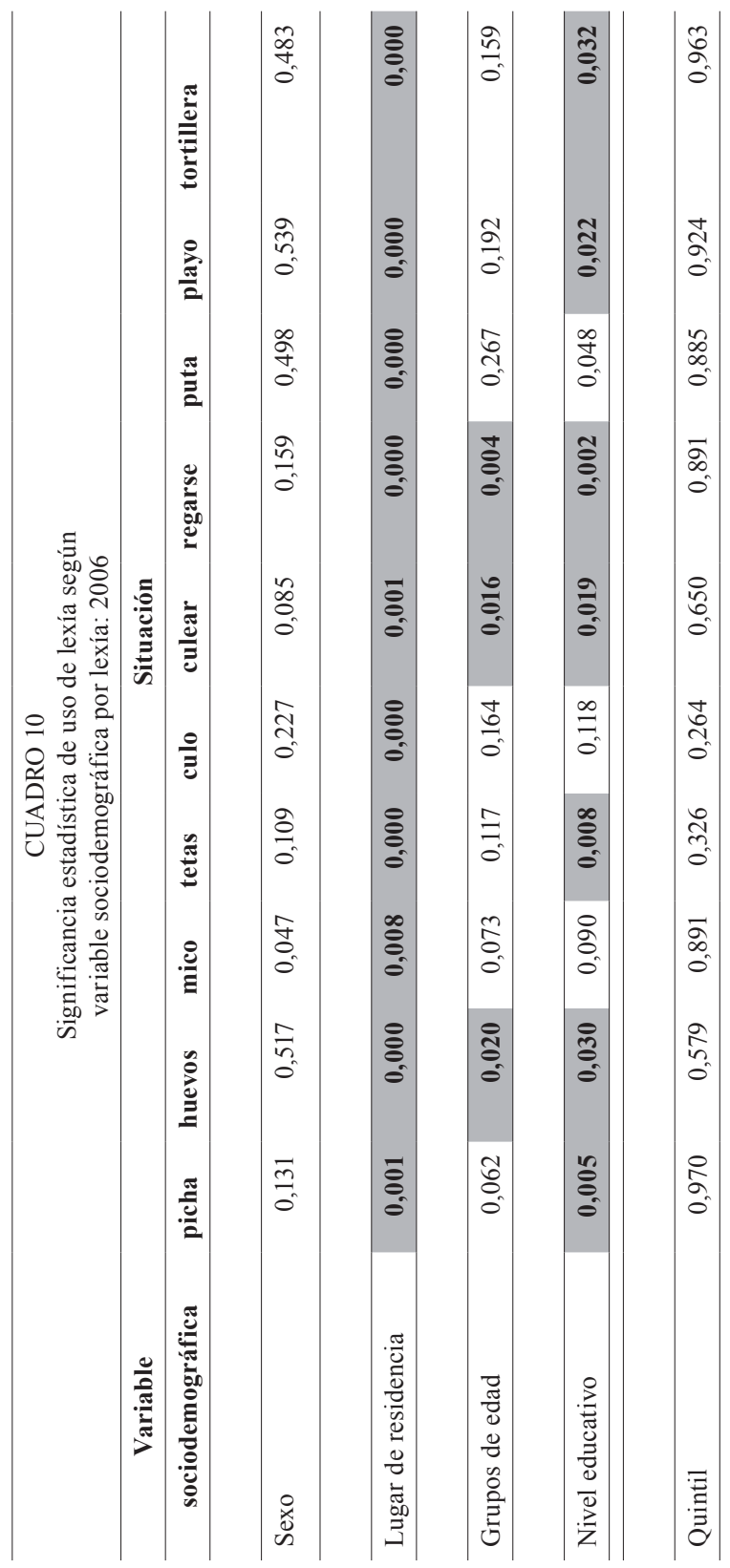


GRÁFICO 2

Porcentaje de uso de lexías según lexía por sexo. 2006.

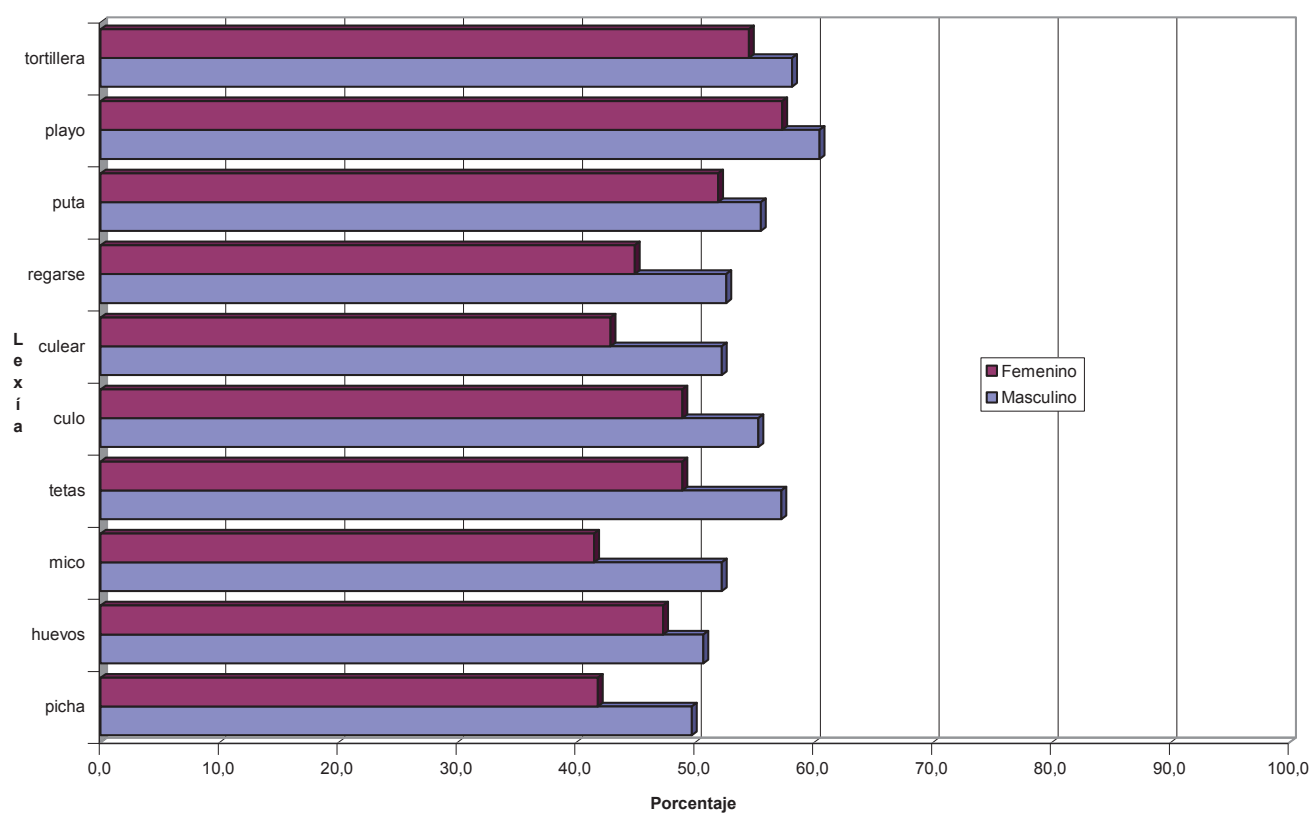

GRÁFICO 3

Porcentaje de uso de lexías según lexía por lugar de residencia. 2006.

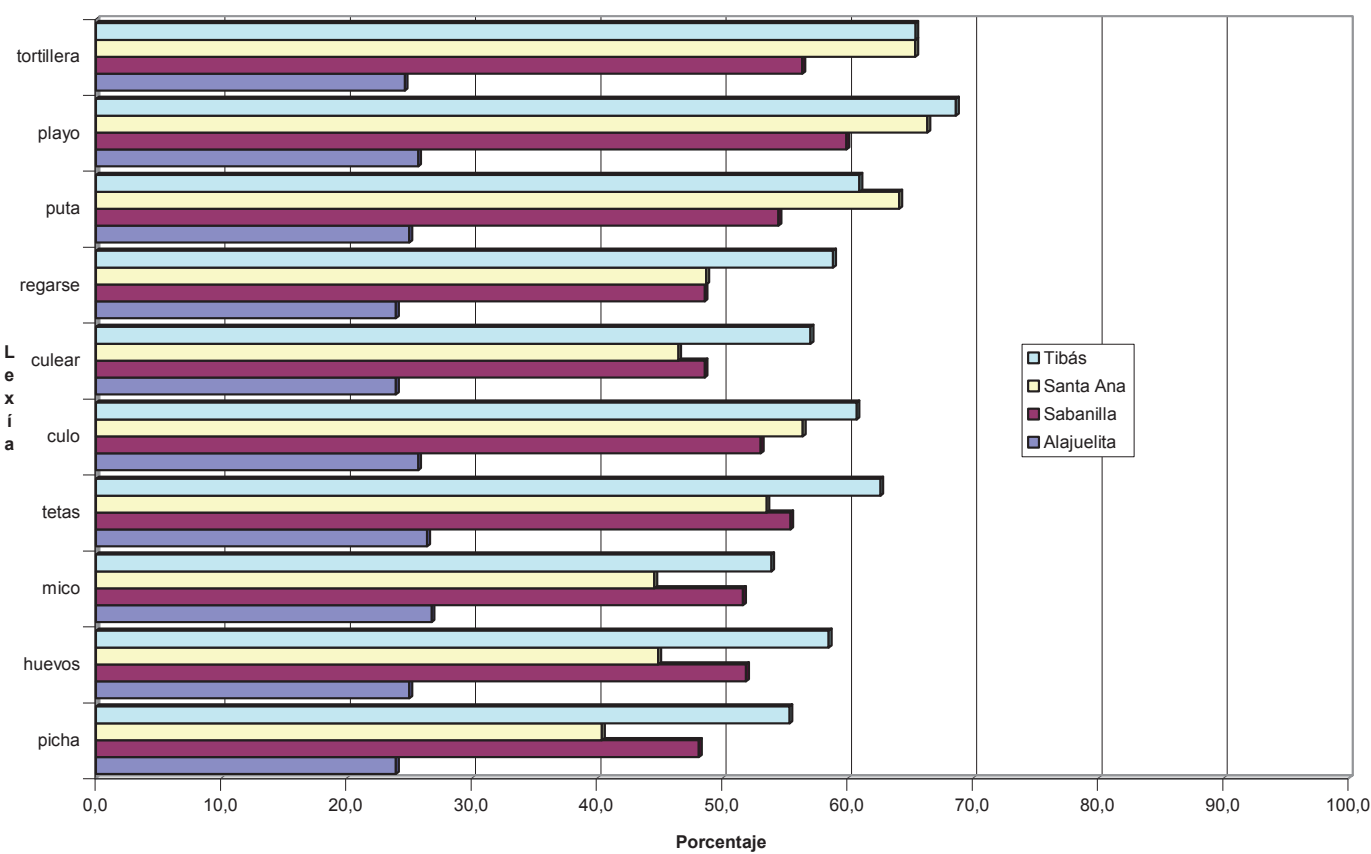


caso, no se puede generalizar el hecho de que las personas de este grupo de edad no conozcan el significado de estas lexías; lo cierto es que las usan menos que el resto de la muestra.

El gráfico 4 muestra las diferencias porcentuales por grupos de edad.

En el gráfico anterior se observa con claridad que las lexías culear y regarse se presentan en un porcentaje mucho menor en el grupo de 55 y más que los otros grupos de edad. La lexía huevos hay que observarla en proporción respecto de las demás. A simple vista, pareciera no ser significativa. Si se observa con atención esta lexía respecto de otros grupos de edad, tiene una frecuencia mucho mayor en el grupo de los 45 a 54 años que en las otras lexías cercanas como picha o mico, que presentan frecuencias menores de este grupo de edad.

Se observa un mayor uso de casi todas las lexías en el grupo de 45 a 54 años, con excepción de puta $(56,4 \%$ en este grupo y $56,6 \%$ en el grupo de 35 a 44), con una diferencia mínima y tetas, también con una diferencia mínima $(57,8 \%$ para el grupo de 45 a 54 , y de $58,3 \%$ para el de 35 a 44).

El grupo de 35 a 44 años sigue al de 45 a 54 en mayor uso en las siguientes lexías: tortillera, culo, mico, huevos.

El grupo menor, de menos de 35 años, también sigue al de 45 a 54 en mayor uso en las siguientes: picha, culear, regarse y playo.

Por edad, el grupo de 45 va 54 presenta el mayor uso de las lexías y el de 55 años y más, el menor uso de lexías. Entre estos dos límites, los grupos de edad de menos de 35 y de 34 a 44 años registran un uso semejante del tabú, considerable pero no mayoritario.

Así pues, la mayor diferencia se observa respecto de los grupos de edad de 55 y más, principalmente, y de 45-54 años.

GRÁFICO 4

Porcentaje de uso de lexías según lexía por grupo de edad. 2006.

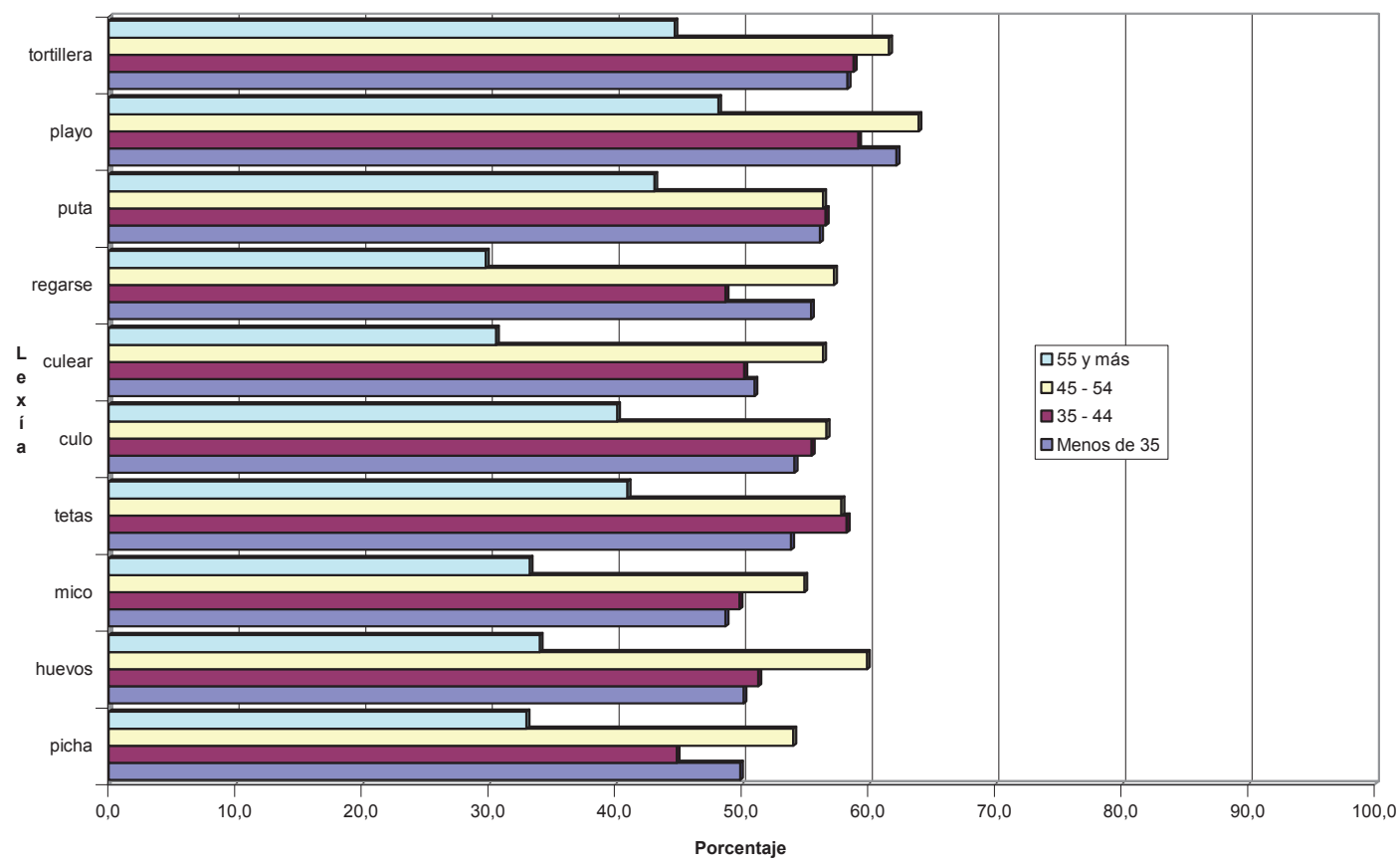


Por nivel educativo, se presenta diferencia significativa en siete de las diez lexías, a saber, picha $(\mathrm{p}=0,005)$, huevos $(\mathrm{p}=0,030)$, tetas $(\mathrm{p}=$ $0,008)$, culear $(P=0,019)$, regarse $(p=0,002)$, playo $(\mathrm{p}=0,022)$ y tortillera $(\mathrm{p}=0,032)$. Las que no mostraron diferencia significativa son mico, culo y puta.

En la lexía picha, se puede observar que las frecuencias más bajas de uso están en la primaria incompleta $(27,2 \%)$ y luego en la primaria completa (36\%). Posteriormente, a partir de la secundaria incompleta, se elevan los porcentajes hasta universitaria incompleta, para reducirse luego en la universitaria completa, con excepción de tetas, que es una de las lexías que presenta un uso más frecuente en la universitaria completa que entre todos los grupos de edad $(65,8 \%)$. Con las otras lexías cuya diferencia es significativa, se observa el mismo patrón.

Para huevos, se observa una diferencia porcentual significativa de $32,1 \%$ en la primaria incompleta, frente a un $61,5 \%$ de uso en la universitaria incompleta. Para tetas, como se observó en el párrafo anterior, la diferencia es significativa pero respecto de la universitaria completa, que es el grupo donde esta lexía registra su porcentaje de uso más alto, $65,8 \%$, seguido muy de cerca por la universitaria incompleta, $64,7 \%$, frente al poco uso, comparativamente, de la primaria incompleta, $31,5 \%$. Para culear, la diferencia entre el uso del grupo de la primaria incompleta, $28,8 \%$, y el de la universitaria incompleta, $60,8 \%$, es muy alta. Con regarse, ocurre algo similar con una diferencia aún más distante entre estos dos grupos, $28,3 \%$ en la primaria incompleta, y $65,4 \%$ en la universitaria incompleta.

Para playo, aunque el porcentaje de uso de esta lexía crece en todos los grupos de edad, el uso en la universitaria incompleta es significativamente alto, $72,6 \%$, frente a un uso de $42,9 \%$ en la primaria incompleta. Así, aunque la lexía mayormente usada en la primaria incompleta es esta, en la universitaria

GRÁFICO 5

Porcentaje de uso de lexías según lexía por nivel educativo. 2006.

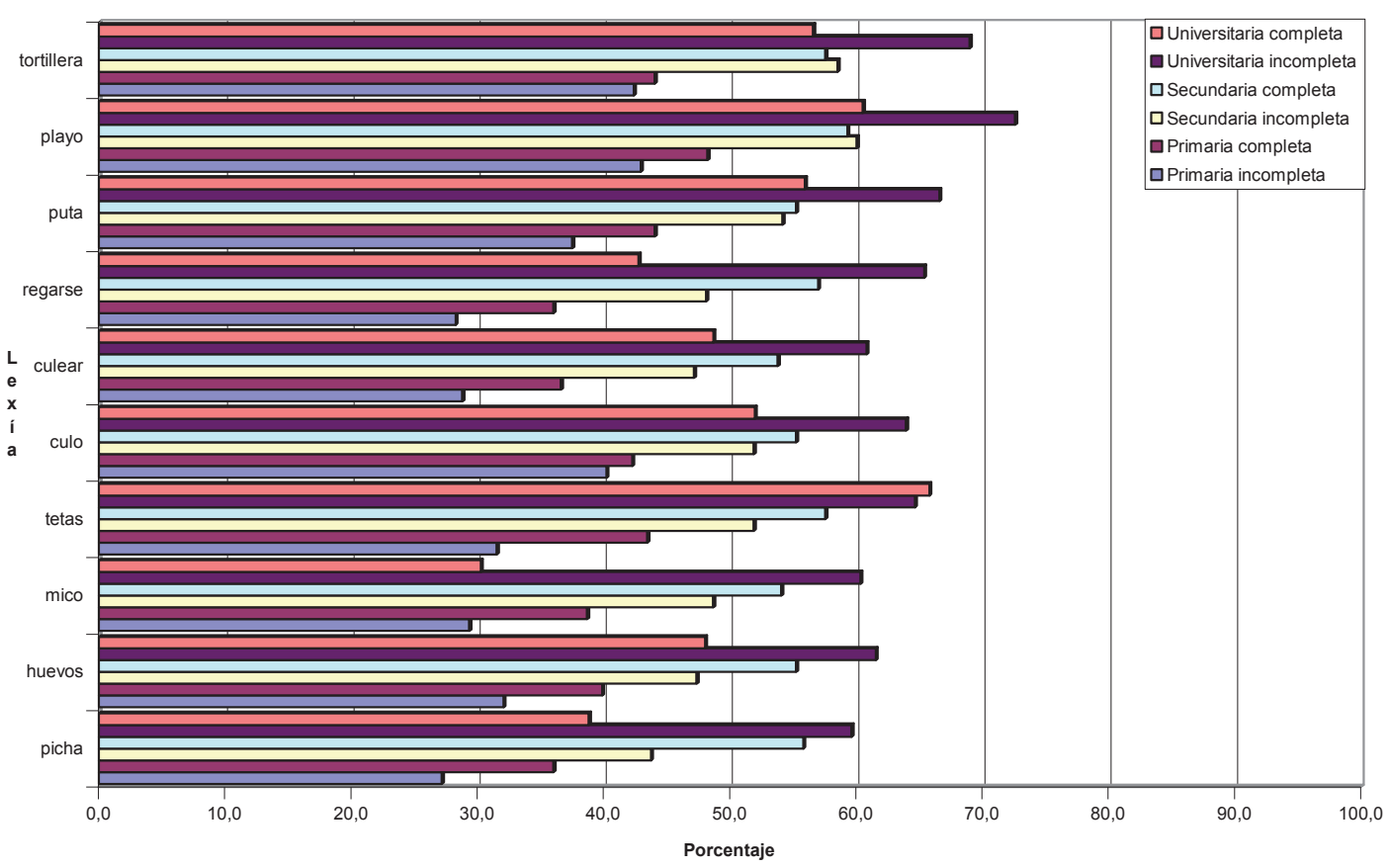


incompleta también resulta la más usada y registra el porcentaje más alto de uso de todas las lexías en todos los grupos de nivel educativo. Por último, tortillera sigue de cerca los resultados de playo. Aun cuando es usada con alta frecuencia en la primaria incompleta, $42,4 \%$, el uso en la universitaria incompleta asciende a una diferencia significativa de un $69 \%$.

En general se puede observar que, con excepción de la lexía tetas, en todas las otras hay una frecuencia de uso significativamente mayor en este grupo educativo que en el resto. El grupo que le sigue no muy de cerca en frecuencia es el de la secundaria completa en la mayoría de las lexías, como en picha, huevos, mico, culo, culear y regarse. Solamente en dos casos la frecuencia de universitaria completa lo secunda, en playo y en puta, y una vez la de secundaria incompleta en playo. En solo un caso, tetas, el grupo de universitaria completa $(65,8 \%)$ supera al de universitaria incompleta $(64,7 \%)$, que lo secunda en este mismo grupo.

Para observar la relación entre las lexías por nivel educativo, se presenta el gráfico 5.

Al contrario de los casos cuya diferencia de uso es significativa, se observa en el gráfico que para mico, los porcentajes no se presentan tan distantes unos de otros y que en el grupo de universitaria incompleta es de las lexías con menor frecuencia de uso $(60,3 \%)$. De este modo, su porcentaje se acerca más a los otros.

En el caso de culo la situación difiere, puesto que es la primaria incompleta el grupo que presenta un ascenso en la frecuencia de uso de esta lexía, $40,2 \%$, frente a un $63,9 \%$ de uso máximo en la universitaria incompleta. Esto hace que los porcentajes se acerquen y que su diferencia porcentual no sea significativa.

En el caso de la lexía puta sucede algo semejante que para mico, pues en la primaria incompleta se registra un porcentaje un tanto elevado de esta lexía (37,5\%), lo cual acerca el porcentaje respecto de la universitaria incompleta $(66,6 \%)$.

Por quintil (nivel socioeconómico), no se percibieron diferencias significativas. Las diferencias porcentuales entre un quintil y otro son mínimas a lo largo de todas las lexías.
Por ejemplo, para la lexía playo, el quintil solo aporta una diferencia de un $5,4 \%$ entre la mayor frecuencia (quintil IV, 58,8\%) y la menor frecuencia (quintil I, 53,4\%). Es interesante, sin embargo, que aunque no haya diferencia significativa, los porcentajes de uso mayores de todas las lexías se encuentran en los quintiles IV y V; es decir, en los más altos niveles socioeconómicos.

\subsection{Análisis de lexías por situación según variables sociodemográficas}

La situación comunicativa es una de las variables lingüísticas fundamentales en este estudio. El estudio de la significancia estadística a este respecto, se muestra a continuación en el siguiente cuadro:

En la variable demográfica sexo, el uso de las lexías resultó significativa en la situación 7. Usted hablando con personas de confianza durante un juego de mesa $(\mathrm{p}=0,037)$.

Por la diferencia en los porcentajes del cuadro 30 , es observable que ambos sexos presentan un uso mucho mayor de las lexías en las situaciones informales, a saber, 1,2,3 y 7, y un notable descenso en su uso en las situaciones formales. En el siguiente gráfico se pueden observar, en forma comparativa, las frecuencias de uso por situación entre ambos sexos.

Como se puede observar en el gráfico 6 , por sexo, los hombres presentan un uso mayor que las mujeres de todas las lexías en todas las situaciones presentadas. La situación que resultó con diferencia significativa, la 7 , presenta una frecuencia de $67,1 \%$ en hombres y de $56,1 \%$ en mujeres. Es la que presenta una mayor variabilidad.

La situación que presenta más uso de las lexías, sin embargo, es la 1 (73,9\% hombres; $60,6 \%$ mujeres); seguidamente la 7 ; luego la 3 $(64,3 \%$ hombres; $56,5 \%$ mujeres) y después la 2 (61,8\% hombres; 59,9\% mujeres), aunque el porcentaje de mujeres es ligeramente mayor que el de la situación 3 .

Todas estas frecuencias corresponden a las situaciones informales, tal y como se esperaba. 
CUADRO 11

Significancia estadística de uso de lexía según variable sociodemográfica por situación

\begin{tabular}{|c|c|c|c|c|c|c|c|c|}
\hline \multirow{2}{*}{$\begin{array}{l}\text { Variable } \\
\text { sociodemográfica }\end{array}$} & \multicolumn{8}{|c|}{ Situación } \\
\hline & 1 & 2 & 3 & 4 & 5 & 6 & 7 & 8 \\
\hline Sexo & 0,080 & 0,723 & 0,133 & 0,610 & 0,346 & 0,503 & $\mathbf{0 , 0 3 7}$ & 0,306 \\
\hline Lugar de residencia & 0,000 & 0,000 & 0,000 & 0,054 & 0,022 & 0,074 & 0,000 & 0,000 \\
\hline Grupos de edad & 0,004 & 0,003 & 0,004 & 0,337 & 0,375 & 0,521 & 0,001 & 0,407 \\
\hline Nivel educativo & 0,012 & 0,020 & 0,004 & 0,027 & 0,033 & 0,070 & 0,009 & 0,075 \\
\hline Quintil & 0,675 & 0,967 & 0,762 & 0,454 & 0,611 & 0,915 & 0,595 & 0,885 \\
\hline
\end{tabular}

GRÁFICO 6

Porcentaje de uso de lexías según situación por sexo. 2006.

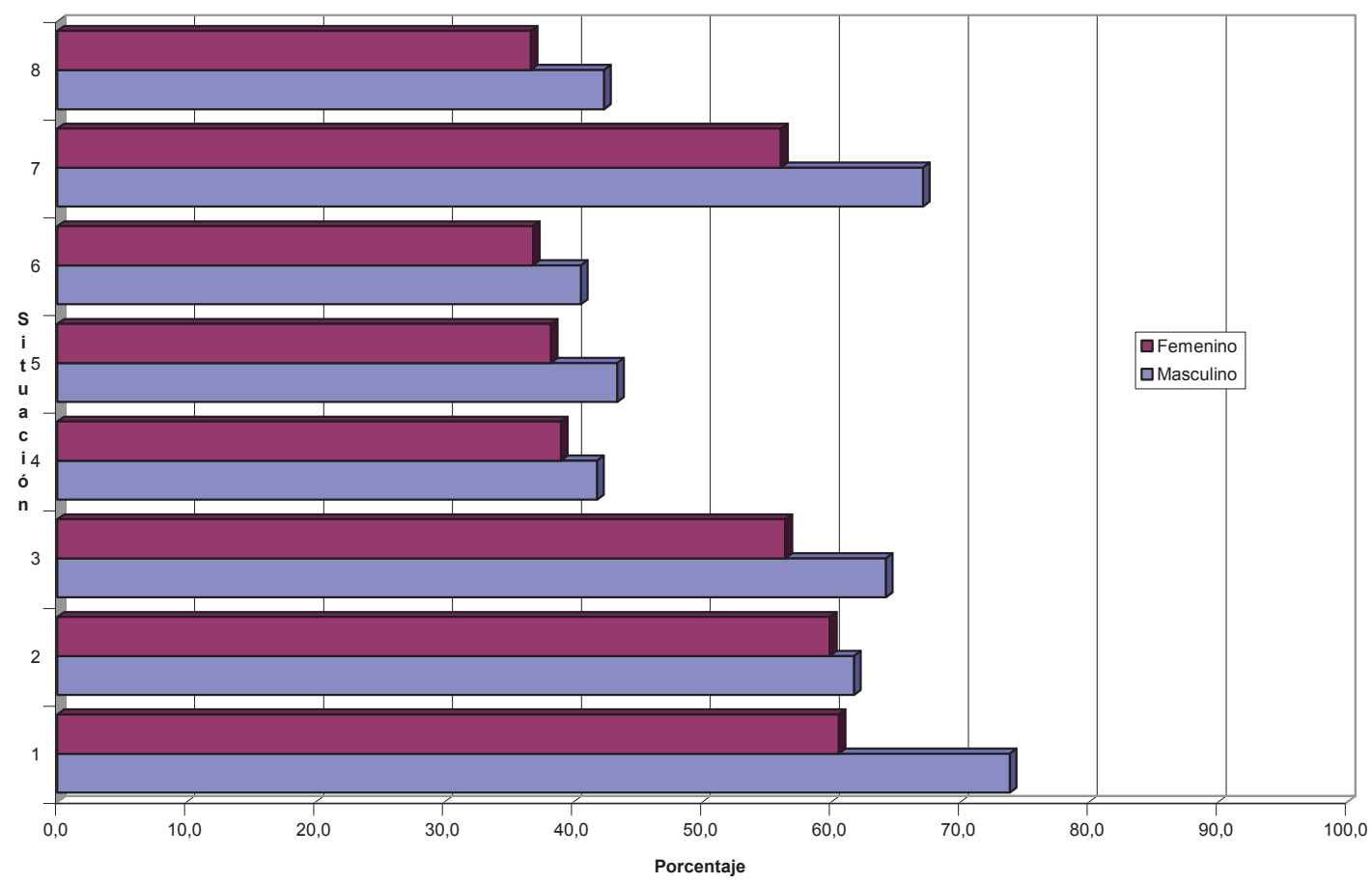


Respecto de las situaciones formales, el orden de de mayor a menor frecuencia es el siguiente: Situación 5 (43,4\% hombres; 38,3\% mujeres); seguidamente la situación $8 \quad(42,4 \%$ hombres; $36,7 \%$ mujeres); luego la situación 4 (41,9\% hombres; $39,1 \%$ mujeres) y, por último, la situación 6 es la menos frecuente por sexo (40,6\% hombres; $36,9 \%$ mujeres).

Por lugar de residencia, los porcentajes son muy interesantes, pues Alajuelita, cantón que a su vez presenta el nivel socioeconómico más bajo, según quintil de ingreso, también presenta los porcentajes más bajos de uso de lexía por situación.

Los porcentajes de uso por situación son bastante similares, y en este cantón se desdibujan un poco las diferencias por situación formal o informal, ya que entre la situación 1 , más informal $(30,0 \%)$ y la más formal, la $4,(23,1 \%)$ hay una diferencia de solo un $6,9 \%$. En los otros lugares de residencia, las lexías se presentan en una proporción mucho mayor por situación, por lo que se puede observar, que hay diferencia estadísticamente significativa en esta variable.
De hecho, existe diferencia significativa en la mayoría de las lexías, a saber, en las situaciones $1,(\mathrm{p}=0,000) 2,(\mathrm{p}=0, .000) 3,(\mathrm{p}=0,000) 5$, $(\mathrm{p}=0,022) 7,(\mathrm{p}=0,000)$ y $8(\mathrm{p}=0.000)$. Las situaciones en que no se revelan diferencias significativas por lugar de residencia son la 4 (p $=0,054)$ y la $6(\mathrm{p}=0,074)$.

El siguiente gráfico muestra las diferencias de lexías por situación según lugar de residencia.

Como se puede observar, Alajuelita es el cantón que menos lexías tabuizadas usó por situación, aportando un menor uso en casi todas las situaciones excepto en la 4 y la 6 las cuales, al ser situaciones formales, presentan reducción en el uso de las lexías en todos los lugares; posteriormente sigue Santa Ana, con un menor uso de las lexías en las situaciones informales, pero en las situaciones formales 4, 5, 6 y 8 , se usaron más que en Sabanilla; posteriormente, Sabanilla usa más las lexías que Santa Ana en las situaciones informales, y Tibás, presenta un uso mayor de las lexías en todas las situaciones. Esto indica que, conforme los lugares de procedencia

\section{GRÁFICO 7}

Porcentaje de uso de lexías según situación por residencia. 2006.

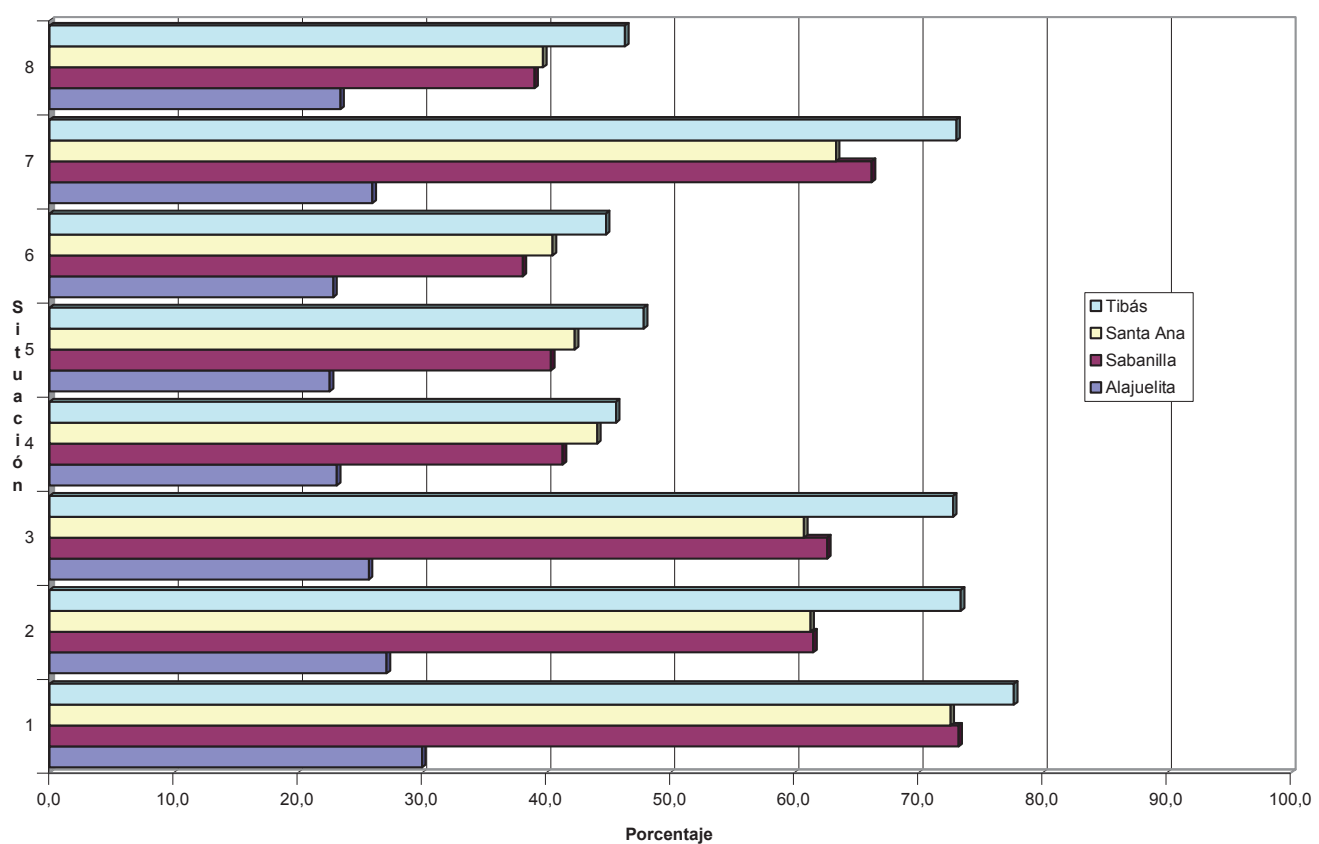


presentan más población en los quintiles más bajos, así las lexías tienen un uso menor. Es decir, a mayor cantidad de población en los quintiles más bajos, menor uso de lexías se registra.

Por grupos de edad, se presenta diferencia significativa en todas las situaciones informales, a saber $1(\mathrm{p}=0,037) ; 2(\mathrm{p}=0,037)$; $3(\mathrm{p}=0,004)$, y $7(\mathrm{p}=0,001)$. El grupo de 55 años y más presenta un uso mucho menor de las lexías en todas las situaciones, si se compara con cualquiera de los otros grupos.

Por ejemplo, en el orden de informal a formal que se tiene planteado, la situación 1 muestra en este grupo un $48 \%$ de uso, frente a más de $70 \%$ de los otros; la situación 7 muestra un $32,3 \%$, frente a más de $39 \%$ de 1 os otros grupos; la situación 2 muestra un 41,1\%, frente a más de $65 \%$ de los demás; la situación 3 muestra un $41,7 \%$, frente a más de un $63 \%$ en los otros grupos; la situación 5 presenta un
$33,0 \%$, frente a más del $40 \%$ de los demás; la situación 4 muestra un 33,0\% frente a más del $39 \%$ de los demás; la situación 8 presenta un $32,3 \%$, frente a más del $39 \%$ de los otros, y la situación 6 presenta un 32,3\%, frente a más de un $36 \%$ de los demás grupos.

El gráfico 8 muestra las lexías por situación según edad.

Como se muestra en el gráfico anterior, el grupo generacional de 55 años y más, muestra el menor uso de las lexías en todas las situaciones. Por el contrario, el grupo etario que más las utiliza en casi todas las situaciones, excepto en la 1 y en la 3, es el grupo de 45 a 54 años. En medio de estas dos generaciones, el grupo de menos de 35 años y el de de 35 a 45 años presentan frecuencias semejantes, con excepción de las situaciones 3 y 1 , cuya mayor frecuencia corresponde al primer grupo, de menos de 35, en la situación 3, y al segundo grupo, de 35 a 44, en la situación 1. Es decir, a mayor edad

GRÁFICO 8

Porcentaje de uso de lexías según situación por grupos de edad. 2006.

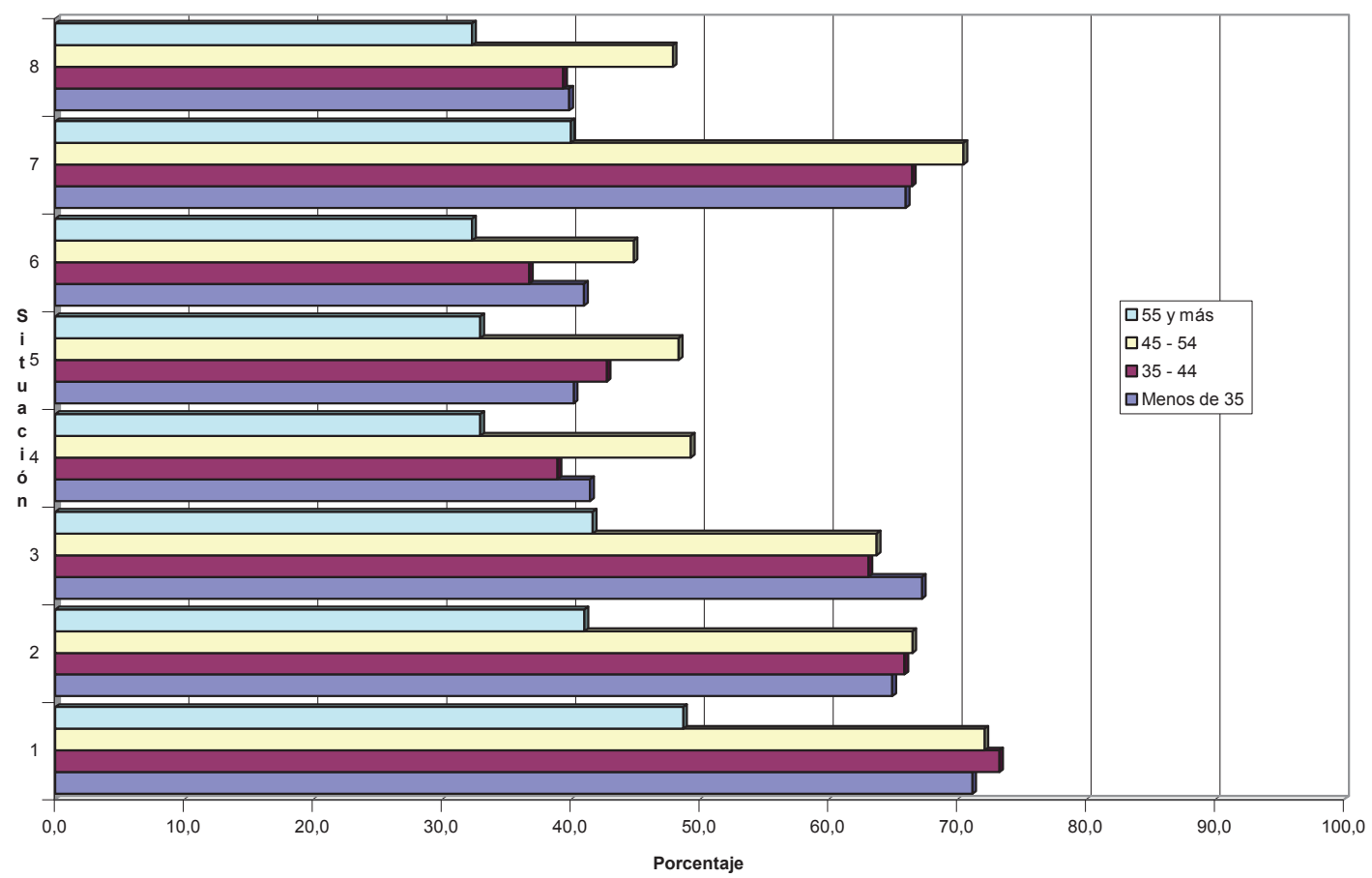


mayor uso de las lexías tabú hasta los 55 años; luego, a partir de los 55 y más, su uso disminuye considerablemente.

Por nivel educativo, se encontró diferencia significativa en las situaciones 1 $(\mathrm{p}=0,012) ; 2(\mathrm{p}=0,020) ; 3(\mathrm{p}=0,004) ; 4$ $(\mathrm{p}=0,027) ; 5(\mathrm{p}=0,033)$, y $7(\mathrm{p}=0,009)$. Se muestra que los entrevistados, a partir de los que han cursado secundaria incompleta, y hasta universitaria completa, muestran un porcentaje de uso mayor de las lexías tabú en todas las situaciones que los que han cursado solo primaria incompleta o completa.

En todas las situaciones se da una diferencia que va entre el $10 \%$ y el $30 \%$ respecto de la primaria incompleta o completa y los niveles educativos más elevados a partir de secundaria incompleta.

Entre los niveles educativos medio y elevado, los grupos que muestran menor uso de las lexías son el grupo de universitaria completa en la mitad de las situaciones $(1,4,7,8)$ y el de secundaria incompleta en la otra mitad de las situaciones $(2,3,5,6)$.

Por otro lado, el grupo que presenta un mayor uso de las lexías por situación es el de universitaria incompleta, con un porcentaje de uso de lexías mayor en todas las situaciones.

Para observar las diferencias entre las lexías por situación según nivel educativo, se presenta el siguiente gráfico.

El gráfico 9 muestra que, en términos generales, el nivel de primaria incompleta presenta un menor uso de las lexías a lo largo de todas las situaciones, seguido por el nivel de primaria completa, que presenta un uso un tanto mayor de las lexías en todas las situaciones, pero igualmente bajo en comparación con los niveles educativos medio y universitario.

El grupo de educación universitaria incompleta, por el contrario, registró un uso mayor de las lexías en todas las situaciones,

\section{GRÁFICO 9}

Porcentaje de uso de lexías según situación por nivel educativo. 2006.

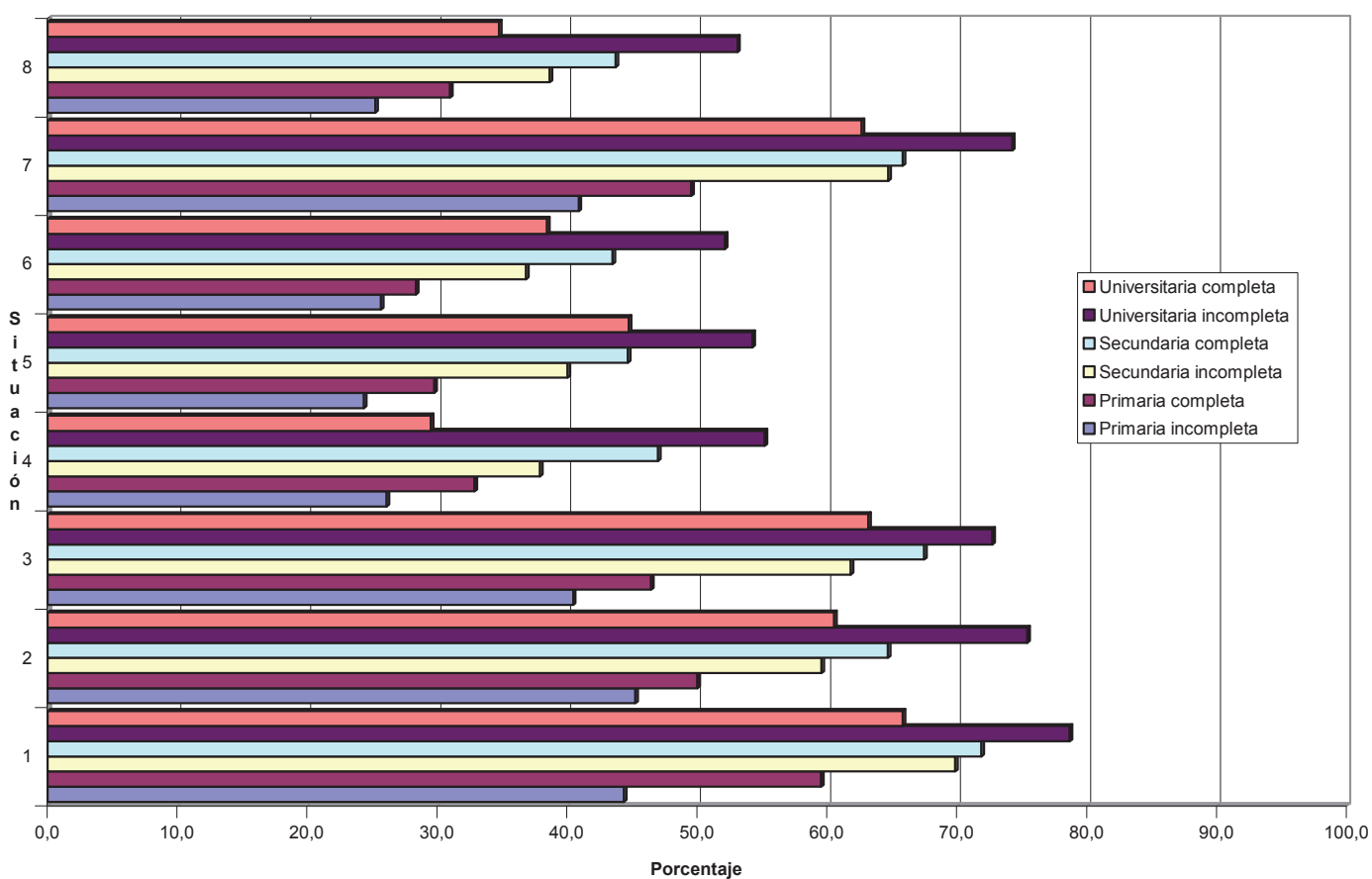


seguido, igualmente en todas las situaciones, por la educación secundaria completa. El nivel de universitaria completa y el de secundaria incompleta se reparten las frecuencias intermedias en las situaciones señaladas anteriormente.

Por situación socioeconómica, el análisis del quintil de ingresos no ha aportado diferencias significativas, como ya se ha mencionado. El quintil muestra que a mayor formalidad en las situaciones hay una disminución porcentual importante respecto del uso de las lexías a lo largo de todos los quintiles, lo cual corrobora que la situación es fundamental en el uso mayor o menor de las lexías, según sea el caso.

\subsection{Síntesis general}

El presente estudio, que ha tomado dos estilos lingüísticos (formal e informal) en cuatro situaciones comunicativas para cada uno de ellos, ha explorado el uso del léxico sexual tabuizado en dichas situaciones con base en cinco variables sociodemográficas: el sexo, la edad, el nivel de instrucción, el lugar de residencia y el quintil socioeconómico. Respecto de este último, se concluyó que la mayor concentración de población se encuentra en los quintiles socioeconómicos más bajos, a saber, en los cantones de Alajuelita y Santa Ana, mientras que en los cantones de Sabanilla y Tibás, se concentra la población de los quintiles de ingresos más altos.

En general, el promedio de uso del tabú en esta investigación es del 51,4\%.

De acuerdo con los resultados de esta investigación, el léxico tabuizado que se relaciona con las partes del cuerpo y con las acciones de tipo sexual se registra con menor frecuencia, mientras el relacionado con la conducta sexual se utiliza con mayor frecuencia, a saber, puta, playo y tortillera dicen ser las más usadas, y mico y picha, las menos usadas.

Por estilo en las situaciones formales, como era de esperar, el uso del tabú es menor, del $40,02 \%$, mientras que en las situaciones informales, el porcentaje de uso del tabú es del $62,75 \%$. Se encontró diferencia estadísticamente significativa en situaciones formales por lugar de residencia, puesto que en Alajuelita se usa significativamente menos el tabú, y en Tibás, se da el mayor uso.

El las situaciones informales se encontró diferencia significativa por lugar de residencia, grupos de edad nivel educativo; es decir, hay un menor uso del tabú también en Alajuelita en estas situaciones, al igual que el grupo de 55 años y más y el nivel educativo más bajo. En general, a partir de entrevistados con nivel de secundaria, el supuesto uso del tabú aumenta considerablemente en ambos estilos. En el nivel formal, hay mayor uso del tabú también conforme aumenta el nivel educativo, aunque no se encontró diferencia significativa.

El estilo según sexo no presenta diferencias significativas, aunque las mujeres dicen emplear el tabú menos que los hombres.

Respecto del análisis de lexías por las variables sociodemográficas, se concluye lo siguiente:

Por sexo no se encontró diferencia estadísticamente significativa en el uso de las lexías; si bien en los hombres se observa un mayor uso de ellas que en las mujeres. En las situaciones, sin embargo, se observa una diferencia significativa por sexo en la situación 7. Usted hablando con personas de confianza durante un juego de mesa, en la que las mujeres usan las lexías tabuizadas significativamente menos que los hombres. En las demás situaciones no se encontró diferencia estadísticamente significativa en el uso de las lexías, pero en los hombres también se observa un mayor uso de ellas que en las mujeres. La situación que usa más lexías tabuizadas por sexo es la 1. Usted hablando en broma con un amigo/a íntimo a solas.

Por lugar de residencia, la diferencia significativa se da en todas las lexías. El cantón de Alajuelita exhibe un uso significativamente menor de todas las lexías en general. El cantón que exhibe una frecuencia mayor de uso es Tibás. Por situación, existe diferencia significativa en la mayoría de las lexías en todas las situaciones excepto en la 4. Usted en una entrevista de trabajo, y en la 6 . Usted en una entrevista por 
televisión, ambas formales y de tipo entrevista. Igualmente, Alajuelita es el cantón que menos lexías tabuizadas usó por situación, y de nuevo, Tibás presentó un uso mayor de las lexías en todas las situaciones.

Por edad se encontró diferencia significativa en las lexías huevos culear y regarse. Estas presentan porcentajes de uso mucho más bajos en el grupo mayor de 55 años. El grupo de 45 a 54 presenta el mayor uso de las lexías y el de 55 años y más, el menor uso. Entre estos dos límites, los grupos de edad de 25 a 35 años y de 36 a 44, registran un uso semejante del tabú; este es considerable, pero no mayoritario. Por situación, se presenta diferencia significativa en todas las situaciones informales.

También, el grupo de 55 años y más presenta un uso mucho menor de las lexías en todas las situaciones y el grupo de 45 a 54, exhibe la mayor frecuencia. Igualmente, los otros dos grupos de edad anteriores registran un uso semejante del tabú, considerable pero no mayoritario.

Por nivel educativo, se presenta diferencia significativa en siete de las diez lexías, picha, huevos, tetas, culear, regarse, playo y tortillera.

En general, hay una frecuencia de uso de las lexías significativamente mayor en el grupo universitaria incompleta. El grupo que le sigue no muy de cerca en frecuencia es el de la secundaria completa en la mayoría de las lexías. Las frecuencias más bajas de uso de todas las lexías se dan en las personas con la primaria incompleta y luego en las de primaria completa. Por situación, se encontró diferencia significativa en todas las situaciones informales $y$ en las formales 4. usted en una entrevista de trabajo, y 5. usted en una declaración frente a un tribunal de justicia.

Por quintil socioeconómico, no se obtuvo diferencia significativa ni por lexía ni por situación. Los porcentajes de uso mayores de todas las lexías se encuentran en los quintiles IV y $\mathrm{V}$, en los más altos niveles socioeconómicos.
En conclusión, el orden en las variables sociodemográficas de acuerdo con la frecuencia en que mostraron diferencia significativa tanto por lexía como por estilo son, en primer lugar, el lugar de residencia, en segundo lugar, el nivel educativo, en tercer lugar, la edad, en cuarto lugar el sexo y, en quinto lugar, el quintil de ingresos.

Uno de los mayores aportes de esta investigación es el hecho de haber tomado en cuenta la variable lugar de residencia, la cual no es frecuente que se utilice en los estudios sociolingüísticos habituales, y que aquí demostró ser la variable sociodemográfica más significativa de todas las que son relevantes para el supuesto uso del tabú lingüístico.

Por otro lado, tratar como variables diferentes el nivel educativo y el quintil de ingresos resultó importante puesto que, a pesar de que el quintil no arrojó resultados significativos, sí mostró que a menor quintil de ingresos hay también menor nivel instrucción formal $\mathrm{y}$, a su vez, a mayor cantidad de población en los quintiles más bajos, se registra un menor uso del tabú lingüístico. Esto es interesante porque al cruzar las variables, los quintiles más bajos de ingresos, congruentes con los niveles más bajos de escolaridad y menor uso del tabú, se encuentran en el cantón más pobre ${ }^{5}$ de los cuatro, a saber, Alajuelita. Por otro lado, el mayor uso del tabú lingüístico se encuentra, a su vez, en el cantón con mayor nivel socioeconómico, Tibás.

\section{Notas}

1. Cf. Calvo Shadid (2011).

2. Según López Morales (1990).

3. La presente investigación replica los estudios de López Morales (1990) y Martínez Valdueza (1995).

4. Alajuelita se erigió como cantón el 2 de junio de 1909, bajo la administración del Lic. Cleto González Víquez.

5. En el sentido de que muestra mayor cantidad de población en los primeros quintiles de ingresos. 


\section{Bibliografía}

Calvo Shadid, A. El tabú lingüístico en el español de San José: estudio piloto. Revista de Filología y Lingüistica de la Universidad de Costa Rica. Vol. 37 N.1. 2011 (en prensa).

Instituto Nacional de Estadística y Censos (INEC). 2000. Costa Rica: Censo de población. Resultados: http://www. prolades.com/costarica/census $2000 /$ censo2000.htm

Instituto Nacional de Estadística y Censos (INEC). Encuesta de Hogares de
Propósitos Múltiples: http://www.inec. go.cr/Web/Home/GeneradorPagina.aspx

López Morales, H. 1990. Sociolingüística del tabú. El caso de Puerto Rico. Madrid: MS.

Martínez Valdueza, P. 1995. El tabú lingüístico: estudio sociolingüístico de Las Palmas de Gran Canaria. Tesis doctoral inédita. Las Palmas de Gran Canaria: Universidad de Las Palmas.

Quesada Pacheco, M.Á. 1991/2001. Nuevo Diccionario de Costarriqueñismos. Cartago: Editorial Tecnológica de Costa Rica. 
